TRANSACTIONS OF THE

AMERICAN MATHEMATICAL SOCIETY

Volume 363, Number 9, September 2011, Pages 5021-5055

S 0002-9947(2011)05408-5

Article electronically published on March 10, 2011

\title{
GLOBAL HEAT KERNEL ESTIMATES FOR SYMMETRIC JUMP PROCESSES
}

\author{
ZHEN-QING CHEN, PANKI KIM, AND TAKASHI KUMAGAI
}

\begin{abstract}
In this paper, we study sharp heat kernel estimates for a large class of symmetric jump-type processes in $\mathbb{R}^{d}$ for all $t>0$. A prototype of the processes under consideration are symmetric jump processes on $\mathbb{R}^{d}$ with jumping intensity

$$
\frac{1}{\Phi(|x-y|)} \int_{\left[\alpha_{1}, \alpha_{2}\right]} \frac{c(\alpha, x, y)}{|x-y|^{d+\alpha}} \nu(d \alpha)
$$

where $\nu$ is a probability measure on $\left[\alpha_{1}, \alpha_{2}\right] \subset(0,2), \Phi$ is an increasing function on $[0, \infty)$ with $c_{1} e^{c_{2} r^{\beta}} \leq \Phi(r) \leq c_{3} e^{c_{4} r^{\beta}}$ with $\beta \in(0, \infty)$, and $c(\alpha, x, y)$ is a jointly measurable function that is bounded between two positive constants and is symmetric in $(x, y)$. They include, in particular, mixed relativistic symmetric stable processes on $\mathbb{R}^{d}$ with different masses. We also establish the parabolic Harnack principle.
\end{abstract}

\section{INTRODUCTION}

The objective of this paper is to give sharp heat kernel estimates for a large class of symmetric jump-type processes in $\mathbb{R}^{d}$ whose jumping kernels decay exponentially. Discontinuous Markov processes and their associated non-local generators have been of current research interest both in probability theory and in PDE, due to their importance in theory and in applications. See, for example, 4, 12, 13, 5 and the references therein. The transition density $p(t, x, y)$ of a Markov process $X$ (if it exists) is the fundamental solution (also called the heat kernel) of a PDE involving the infinitesimal generator $\mathcal{L}$, whose explicit expression is typically impossible to get. Thus it is a fundamental problem, both in probability theory and in analysis, to obtain sharp estimates of $p(t, x, y)$. When $X$ is a symmetric diffusion on $\mathbb{R}^{d}$ whose infinitesimal generator is a uniformly elliptic and bounded divergence form operator, it is well known that $p(t, x, y)$ enjoys the celebrated Aronson's Gaussian type estimates. When $X$ is a pure jump symmetric process on $\mathbb{Z}^{d}$ or $\mathbb{R}^{d}$ whose jumping kernel is of stable or mixed stable type (that decays polynomially at infinity), sharp estimates on $p(t, x, y)$ have been derived in 4 ( on $\mathbb{Z}^{d}$ ) and in 12, 13, (on

Received by the editors March 23, 2010.

2010 Mathematics Subject Classification. Primary 60J75, 60J35; Secondary 31C25, 31B05.

Key words and phrases. Dirichlet form, jump process, jumping kernel, parabolic Harnack inequality, heat kernel estimates.

The first author's research was partially supported by NSF Grant DMS-0906743.

The second author's research was supported by a National Research Foundation of Korea Grant funded by the Korean Government (2009-0087117).

The second and the third authors' research was partially supported by the Global COE program at the Department of Mathematics, Faculty of Science, Kyoto University. 
$\mathbb{R}^{d}$ and beyond). Moreover, in [13, sharp heat kernel estimates have also been obtained in a finite time interval for a class of symmetric jump-type processes whose jumping kernels decay at the rate $e^{-c|x|}$ as $|x| \rightarrow \infty$. It, in particular, contains relativistic stable processes as a special case. Relativistic stable processes is a class of symmetric Lévy processes whose infinitesimal generators are $m-\left(m^{2 / \alpha}-\Delta\right)^{\alpha / 2}$, where $\alpha \in(0,2)$ and $m>0$. The operator $m-\sqrt{m^{2}-\Delta}$, corresponding to $\alpha=1$, is important in mathematical physics due to its correspondence with the kinetic energy of a relativistic particle with mass $m$; see, for example, 7, 18. In applications, one also encounters a class of pure jump Lévy processes in $\mathbb{R}^{d}$ whose jumping kernels decay at the rate $e^{-c|x|^{\beta}}$ as $|x| \rightarrow \infty$ for $\beta \in(0, \infty]$ other than $\beta=1$, which includes a subclass of tempered stable processes in $\mathbb{R}^{d}$. The latter arises in statistical physics to model turbulence as well as in mathematical finance to model stochastic volatility; see, for example, , 8, 17, 19, 21, 23. In these models, both the small time and large time behavior for the transition density function are important. However, except in some very special case and especially for the large time region, detailed quantitative knowledge on the global behavior of $p(t, x, y)$ for these models has not been available until now. The motivation of this paper is to give sharp global transition density estimates of a class of pure jump symmetric processes (not just Lévy processes) in $\mathbb{R}^{d}$ whose jumping kernels decay at the rate $e^{-c|x|^{\beta}}$ as $|x| \rightarrow \infty$ for all $\beta \in(0, \infty]$.

Throughout this paper, $d \geq 1$. Let $\mathbb{R}^{d}$ be the $d$-dimensional Euclidean space and $d x$ or $\mu_{d}$ be the $d$-dimensional Lebesgue measure in $\mathbb{R}^{d}$. For $x \in \mathbb{R}^{d}$ and $r>0$, let $B(x, r)$ denote the open ball centered at $x$ with radius $r$. For two non-negative functions $f$ and $g$, the notation $f \asymp g$ means that there are positive constants $c_{1}, c_{2}, c_{3}$ and $c_{4}$ so that $c_{1} g\left(c_{2} x\right) \leq f(x) \leq c_{3} g\left(c_{4} x\right)$ in the common domain of definitions for $f$ and $g$. The Euclidean distance between $x$ and $y$ will be denoted as $|x-y|$. For $a, b \in \mathbb{R}, a \wedge b:=\min \{a, b\}$ and $a \vee b:=\max \{a, b\}$. Here and in the following, we use ":=" to denote a definition, which is read as "is defined to be".

We assume that $\phi$ can be expressed as

$$
\phi(r)=\phi_{1}(r) \psi_{1}(r) \quad \text { for } r>0,
$$

where $\psi_{1}$ is an increasing function on $[0, \infty)$ with $\psi_{1}(r)=1$ for $0<r \leq 1$ and there are constants $\gamma_{2} \geq \gamma_{1}>0$ and $\beta>0$ so that

$$
c_{1} e^{\gamma_{1} r^{\beta}} \leq \psi_{1}(r) \leq c_{2} e^{\gamma_{2} r^{\beta}} \quad \text { for every } 1<r<\infty,
$$

while $\phi_{1}$ is a strictly increasing function on $[0, \infty)$ with $\phi_{1}(0)=0, \phi_{1}(1)=1$ and there exist constants $0<c_{1}<c_{2}, c_{3}>0$ and $0<\beta_{1} \leq \beta_{2}<2$ so that

$$
c_{1}\left(\frac{R}{r}\right)^{\beta_{1}} \leq \frac{\phi_{1}(R)}{\phi_{1}(r)} \leq c_{2}\left(\frac{R}{r}\right)^{\beta_{2}} \quad \text { for every } 0<r<R<\infty
$$

and

$$
\int_{0}^{r} \frac{s}{\phi_{1}(s)} d s \leq c_{3} \frac{r^{2}}{\phi_{1}(r)} \quad \text { for every } r>0 .
$$

Remark 1.1. Note that condition (1.3) is equivalent to the existence of constants $c_{4}, c_{5}>1$ and $L_{0}>1$ such that for every $r>0$,

$$
c_{4} \phi_{1}(r) \leq \phi_{1}\left(L_{0} r\right) \leq c_{5} \phi_{1}(r) .
$$


Let $J$ be a symmetric measurable function on $\mathbb{R}^{d} \times \mathbb{R}^{d} \backslash\{x=y\}$ such that

$$
J(x, y) \asymp \frac{1}{|x-y|^{d} \phi(|x-y|)} .
$$

By (1.5), the condition (1.6) is equivalent to

$$
\begin{aligned}
& \kappa_{1}^{-1} \frac{1}{|x-y|^{d} \phi_{1}(|x-y|) \psi_{1}\left(\kappa_{2}|x-y|\right)} \\
& \leq J(x, y) \leq \kappa_{1} \frac{1}{|x-y|^{d} \phi_{1}(|x-y|) \psi_{1}\left(\kappa_{3}|x-y|\right)}
\end{aligned}
$$

for every $(x, y) \in \mathbb{R}^{d} \times \mathbb{R}^{d} \backslash\left\{(x, y) \in \mathbb{R}^{d} \times \mathbb{R}^{d}: x=y\right\}$. Here $\kappa_{1}, \kappa_{2}, \kappa_{3}$ are positive constants.

For $u \in L^{2}\left(\mathbb{R}^{d}, d x\right)$, define

$$
\mathcal{E}(u, u):=\int_{\mathbb{R}^{d} \times \mathbb{R}^{d}}(u(x)-u(y))^{2} J(x, y) d x d y,
$$

and for $\beta>0$,

$$
\mathcal{E}_{\beta}(u, u):=\mathcal{E}(u, u)+\beta \int_{\mathbb{R}^{d}} u(x)^{2} d x .
$$

Let $C_{c}\left(\mathbb{R}^{d}\right)$ denote the space of continuous functions with compact support in $\mathbb{R}^{d}$, equipped with the uniform topology. Define

$$
\mathcal{D}(\mathcal{E}):=\left\{f \in C_{c}\left(\mathbb{R}^{d}\right): \mathcal{E}(f, f)<\infty\right\} .
$$

By [13, Proposition 2.2], $(\mathcal{E}, \mathcal{F})$ is a regular Dirichlet form on $L^{2}\left(\mathbb{R}^{d}, d x\right)$, where $\mathcal{F}:=\overline{\mathcal{D}}(\mathcal{E})^{\mathcal{E}_{1}}$. So there is a Hunt process $Y$ associated with it on $\mathbb{R}^{d}$, starting from quasi-every point in $\mathbb{R}^{d}$ (see [16]). In fact, by Meyer's construction (see, e.g., [13, §4.1]), process $Y$ can be constructed from the pure jump process $X$ whose corresponding jumping kernel is the same as $Y$ but with constant function 1 in place of $\psi_{1}$, by removing jumps of size larger than 1 with suitable rate. From the Hölder continuity and the two-sided estimates for the transition density of $X$ obtained in [13, we can refine the process $X$ and therefore the process $Y$ to start from every point in $\mathbb{R}^{d}$. Furthermore, by (1.6) and [20, Theorem 3.1], $Y$ is conservative; that is, $Y$ has infinite lifetime.

It is not difficult to see that with $J$ satisfying (1.7) we have

$$
\mathcal{F}=\left\{u \in L^{2}\left(\mathbb{R}^{d} ; d x\right): \mathcal{E}(u, u)<\infty\right\} .
$$

We say UJS holds (see [2]) if for a.e. $x, y \in \mathbb{R}^{d}$,

$$
J(x, y) \leq \frac{c}{r^{d}} \int_{B(x, r)} J(z, y) d z \quad \text { whenever } r \leq \frac{1}{2}|x-y| .
$$

Note that UJS holds if $\kappa_{2}=\kappa_{3}$ in (1.7); see Lemma 2.1.

The jumping intensity kernel $J(x, y)$ determines a Lévy system of $Y$, which describes the jumps of the process $Y$ : for any non-negative measurable function $f$ on $\mathbb{R}_{+} \times \mathbb{R}^{d} \times \mathbb{R}^{d}$ with $f(s, x, x)=0$ for all $s>0$ and $x \in \mathbb{R}^{d}$ and stopping time $T$ (with respect to the filtration of $Y$ ),

$$
\mathbb{E}_{x}\left[\sum_{s \leq T} f\left(s, Y_{s-}, Y_{s}\right)\right]=\mathbb{E}_{x}\left[\int_{0}^{T}\left(\int_{\mathbb{R}^{d}} f\left(s, Y_{s}, y\right) J\left(Y_{s}, y\right) d y\right) d s\right] .
$$

(See, for example, [12, Proof of Lemma 4.7] and [13, Appendix A].) 
A prototype of the model considered in this paper is the following. Let

$$
\begin{aligned}
\mathcal{E}(f, f) & =\int_{\mathbb{R}^{d} \times \mathbb{R}^{d}}(f(x)-f(y))^{2} J(x, y) d x d y \\
\mathcal{F} & =\left\{f \in L^{2}\left(\mathbb{R}^{d}, d x\right): \mathcal{E}(f, f)<\infty\right\}
\end{aligned}
$$

where $J(x, y)$ is a symmetric kernel given by

$$
J(x, y)=\int_{\left[\alpha_{1}, \alpha_{2}\right]} \frac{c(\alpha, x, y)}{|x-y|^{d+\alpha} \Phi(|x-y|)} \nu(d \alpha),
$$

where $\nu$ is a probability measure on $\left[\alpha_{1}, \alpha_{2}\right] \subset(0,2), \Phi$ is an increasing function on $[0, \infty)$ with $c_{1} e^{c_{2} r^{\beta}} \leq \Phi(r) \leq c_{3} e^{c_{4} r^{\beta}}$ with $\beta \in(0, \infty)$, and $c(\alpha, x, y)$ is a jointly measurable function that is symmetric in $(x, y)$ and is bounded between two positive constants.

The main result of this paper is the following heat kernel estimates. The inverse function of the strictly increasing function $t \mapsto \phi(t)$ will be denoted by $\phi^{-1}(t)$. In this theorem, constants may depend on $\beta>0$.

Theorem 1.2. Assume that conditions (1.1)-(1.4), (1.7) and UJS hold. Then there is a conservative Feller process $Y$ associated with $(\mathcal{E}, \mathcal{F})$ that can start from every point in $\mathbb{R}^{d}$. Moreover, the process $Y$ has a continuous transition density function $p(t, x, y)$ on $(0, \infty) \times \mathbb{R}^{d} \times \mathbb{R}^{d}$ with respect to the Lebesgue measure, which has the following estimates (see Figures 1 and 2).

(1) When $0<\beta \leq 1$ :

(1.a) (Short time estimates) There are positive constants $c_{1}, c_{2}$ and $C \geq 1$ such that for every $t \in(0,1]$ and $x, y \in \mathbb{R}^{d}$,

$$
\begin{aligned}
& C^{-1}\left(\frac{1}{\phi^{-1}(t)^{d}} \wedge \frac{t}{|x-y|^{d} \phi\left(c_{1}|x-y|\right)}\right) \\
& \leq p(t, x, y) \leq C\left(\frac{1}{\phi^{-1}(t)^{d}} \wedge \frac{t}{|x-y|^{d} \phi\left(c_{2}|x-y|\right)}\right) .
\end{aligned}
$$

(1.b) (Large time estimates) There are positive constants $c_{1}, c_{2}$ and $C \geq 1$ such that for every $t \in[1, \infty)$ and $x, y \in \mathbb{R}^{d}$,

$$
C^{-1} t^{-d / 2} e^{-c_{1}\left(|x-y|^{\beta} \wedge \frac{|x-y|^{2}}{t}\right)} \leq p(t, x, y) \leq C t^{-d / 2} e^{-c_{2}\left(|x-y|^{\beta} \wedge \frac{|x-y|^{2}}{t}\right)} .
$$

(2) When $\beta \in(1, \infty)$ :

(2.a) (Short time estimates) There are positive constants $c_{1}, c_{2}$ and $C \geq 1$ such that

$$
\begin{aligned}
& C^{-1}\left(\frac{1}{\phi_{1}^{-1}(t)^{d}} \wedge \frac{t}{|x-y|^{d} \phi_{1}(|x-y|)}\right) \\
& \leq p(t, x, y) \leq C^{-1}\left(\frac{1}{\phi_{1}^{-1}(t)^{d}} \wedge \frac{t}{|x-y|^{d} \phi_{1}(|x-y|)}\right)
\end{aligned}
$$

for every $t \in(0,1]$ and $|x-y|<1$, and

$$
\begin{aligned}
& C^{-1} t e^{-c_{1}\left(\left(|x-y|\left|\log \frac{|x-y|}{t}\right|^{(\beta-1) / \beta}\right) \wedge|x-y|^{\beta}\right)} \\
& \leq p(t, x, y) \leq C t e^{-c_{2}\left(\left(|x-y|\left|\log \frac{|x-y|}{t}\right|^{(\beta-1) / \beta}\right) \wedge|x-y|^{\beta}\right)}
\end{aligned}
$$

for every $t \in(0,1]$ and $|x-y| \geq 1$. 


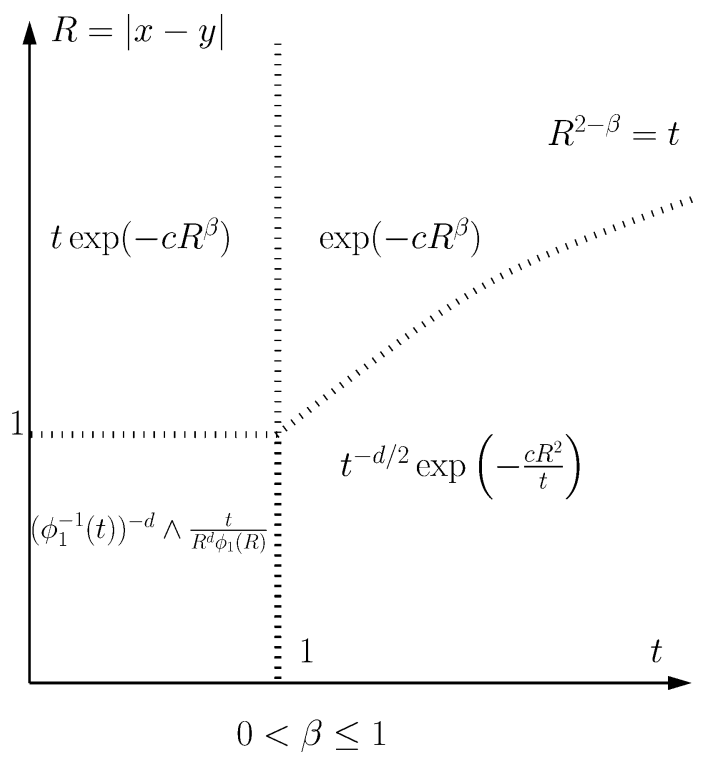

Figure 1

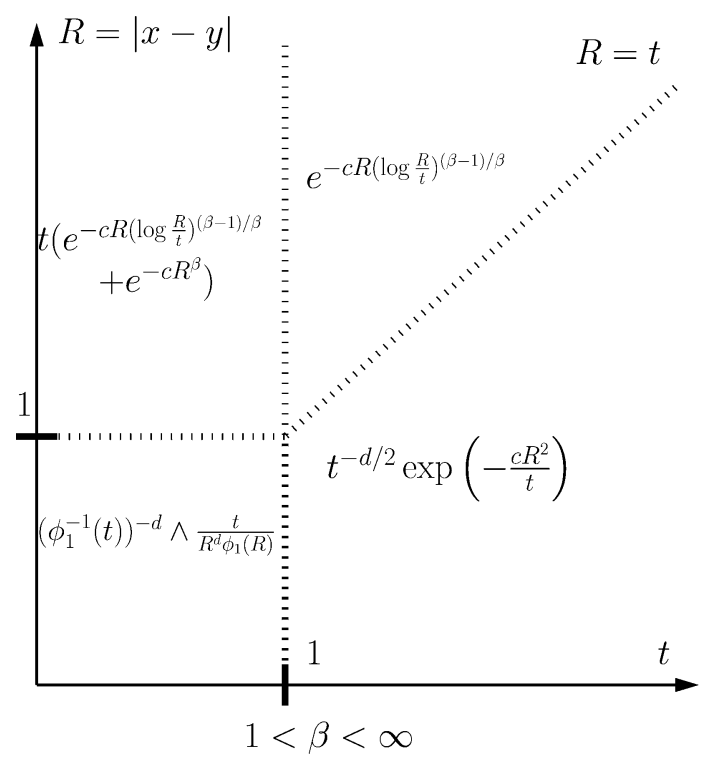

Figure 2

(2.b) (Large time estimates) There are positive constants $c_{1}, c_{2}$ and $C \geq 1$ such that for every $t \in[1, \infty)$ and $x, y \in \mathbb{R}^{d}$,

$$
\begin{aligned}
& C^{-1} t^{-d / 2} e^{-c_{1}}\left(\left(|x-y|\left|\log \frac{|x-y|}{t}\right|^{(\beta-1) / \beta}\right) \wedge \frac{|x-y|^{2}}{t}\right) \\
& \leq p(t, x, y) \leq C t^{-d / 2} e^{-c_{2}\left(\left(|x-y|\left|\log \frac{|x-y|}{t}\right|^{(\beta-1) / \beta}\right) \wedge \frac{|x-y|^{2}}{t}\right)} .
\end{aligned}
$$


Remark 1.3. (i) When $\beta=1$, the short time heat kernel estimates in Theorem 1.2(1.a) is first established in [13, Theorem 1.2].

(ii) Theorem 1.2 shows that there is a phase transition at $\beta=1$ for both the short time and the large time estimates for the transition density function of $Y$.

(iii) Observe that when $\beta>1$, there is a constant $c=c(\beta)>0$ so that for $t \geq 1$ and $|x-y| \geq 1$ (by considering cases $|x-y| \geq 2 t$ and $|x-y|<2 t$ separately),

$$
c|x-y|^{\beta} \geq\left(|x-y|\left|\log \frac{|x-y|}{t}\right|^{(\beta-1) / \beta}\right) \wedge \frac{|x-y|^{2}}{t} .
$$

This explains why $|x-y|^{\beta}$ does not appear in the exponent of the estimates (1.17).

(iv) By the definition (1.1) of $\phi$, when $\beta \in(0,1]$, estimate (1.13) for $t \in(0,1]$ and $|x-y| \geq 1$ is equivalent to the following: there are positive constants $c_{1}, c_{2}$ and $C \geq 1$ so that for every $t \in(0,1]$ and $|x-y| \geq 1$,

$$
C^{-1} t e^{-c_{1}|x-y|^{\beta}} \leq p(t, x, y) \leq C t e^{-c_{2}|x-y|^{\beta}} .
$$

To comprehend estimate (1.14), observe that

$$
|x-y|^{\beta} \wedge \frac{|x-y|^{2}}{t}= \begin{cases}|x-y|^{\beta} & \text { if }|x-y|^{2-\beta} \geq 1, \\ \frac{|x-y|^{2}}{t} & \text { if }|x-y|^{2-\beta}<1 .\end{cases}
$$

(v) When $J(x, y)$ is a function of $|x-y|$, the associated process $Y$ is a symmetric Lévy process. There is a large class of symmetric Lévy processes whose Lévy jumping kernels satisfying the condition (1.7), including relativistic stable processes mentioned at the beginning of this paper. Theorem 1.2, in particular, gives global two-sided sharp estimates for these Lévy processes. The large time heat kernel estimates are new, even for relativistic stable processes. (Short time heat kernel estimates for relativistic stable processes were obtained in [13.) Of course, Theorem 1.2 goes far beyond Lévy processes; it covers a large class of symmetric Markov processes which may not be Lévy and gives the stability results on the heat kernel estimates for this class of symmetric jump processes.

The next theorem deals with the limiting case $\beta \rightarrow \infty$. It extends the heat kernel estimate result in [10, where $\phi_{1}(r)$ is taken to be $r^{\alpha}$ for any $\alpha \in(0,2)$. However, the proof in [10] extends easily to the general $\phi_{1}$ that satisfies the conditions (1.3) and (1.4). So we skip the proof of Theorem 1.4. Note that, from the proof of Lemma 2.1, it is easy to see that UJS holds for $J$ in the next theorem.

Theorem 1.4. Assume that conditions (1.3) and (1.4) hold and $J(x, y) \asymp$ $\frac{1}{|x-y|^{d} \phi_{1}(|x-y|)} \mathbf{1}_{\{|x-y| \leq 1\}}$. Then there is a conservative Feller process $Y$ associated with $(\mathcal{E}, \mathcal{F})$ that starts from every point in $\mathbb{R}^{d}$. Moreover, the process $Y$ has a continuous transition density function on $(0, \infty) \times \mathbb{R}^{d} \times \mathbb{R}^{d}$, which has the following estimates. There are positive constants $c_{1}, c_{2}$ and $C \geq 1$ such that

$$
\begin{aligned}
& C^{-1}\left(\frac{1}{\phi_{1}^{-1}(t)^{d}} \wedge \frac{t}{|x-y|^{d} \phi_{1}(|x-y|)}\right) \\
& \leq p(t, x, y) \leq C^{-1}\left(\frac{1}{\phi_{1}^{-1}(t)^{d}} \wedge \frac{t}{|x-y|^{d} \phi_{1}(|x-y|)}\right)
\end{aligned}
$$


for every $t \in(0,1]$ and $|x-y|<1$,

$$
C^{-1} e^{-c_{1}|x-y| \log \frac{|x-y|}{t}} \leq p(t, x, y) \leq C e^{-c_{2}|x-y| \log \frac{|x-y|}{t}}
$$

for every $t \in(0,1]$ and $|x-y| \geq 1$, and

$$
\begin{aligned}
& C^{-1} t^{-d / 2} e^{-c_{1}\left(\left(|x-y| \log \frac{|x-y|}{t}\right) \wedge \frac{|x-y|^{2}}{t}\right)} \\
& \leq p(t, x, y) \leq C t^{-d / 2} e^{-c_{1}\left(\left(|x-y| \log \frac{|x-y|}{t}\right) \wedge \frac{|x-y|^{2}}{t}\right)}
\end{aligned}
$$

for every $t>1$ and $x, y \in \mathbb{R}^{d}$.

Note that the estimate in (1.20)-(1.21) is the limiting case as $\beta \rightarrow \infty$ in (1.16)(1.17). This indicates that the constant $C$ as well as $c_{1}$ and $c_{2}$ in Theorem 1.2 may be independent of $\beta \in\left[\beta_{0}, \infty\right)$ for every $\beta_{0} \geq 1$. But we are not going to pursue this independence in this paper.

The proof of Theorem 1.2 requires different techniques for different cases, which will be given separately. In fact, some estimates in Theorem 1.2, especially the upper bound estimates, are established for more general jumping kernel $J$ under condition (3.12) rather than under (1.7).

For the upper bounds, the proof of (1.18) is given in Theorem 3.4. The strategy is to first consider the finite range process $Y^{(\lambda)}$, whose jumping kernel is $J(x, y) \mathbf{1}_{\{|x-y| \leq \lambda\}}$, and use Davies' method from [6] to derive an upper bound estimate for the transition density function of $Y^{(\lambda)}$ through carefully chosen testing functions. Here we need to select the value of $\lambda$ in a very careful way that depends on the values of $t,|x-y|$ and $\beta$. The upper bound estimates for the transition density function of $Y$ are then obtained from those of $Y^{(\lambda)}$ through Meyer's construction of $Y$ from $Y^{(\lambda)}$. Specifically, (1.14) is proved in Proposition 3.1 when $|x-y| \leq c$ and in Theorem 3.3 when $|x-y| \geq c$. The upper bounds proof for (1.17) and (1.16) are given in Theorem 3.2 and Theorems 3.4 3.6. respectively. All these are summarized in Theorem 3.6. where the proof of the upper bound estimates in Theorem 1.4 is also given.

For the lower bounds, the proof of (1.18) is given in Theorem 5.3. The strategy is to first derive large time near the diagonal lower bound estimate. For this, we follow an approach from [1, using an enhanced version of the weighted Poincaré inequality which was initially established in 10 and an entropy argument. We next establish the parabolic Harnack principle (PHI in abbreviation) for $Y$. The lower bound off-diagonal estimates on $p(t, x, y)$ are then obtained after certain exit time estimates and transition probability estimates are established. Specifically, (1.14) is proved in Theorem 4.8 and Theorem 5.4. The lower bounds of (1.16) and (1.17) are established in Theorems 5.5, 4.8 and 5.4(i).

Throughout this paper, we use $c_{1}, c_{2}, \cdots$ to denote generic constants whose exact values are not important and can change from one appearance to another. The labeling of the constants $c_{1}, c_{2}, \cdots$ starts anew in the statement of each result. The dependence of the constant $c$ on the dimension $d$ may not be mentioned explicitly. For $p \in[1, \infty]$, we will use $\|f\|_{p}$ to denote the $L^{p}$-norm in $L^{p}\left(\mathbb{R}^{d}, d x\right)$. 


\section{UJS AND EXAMPLES}

Lemma 2.1. Assume that $J$ is given by (1.7) with $\kappa_{2}=\kappa_{3}$. Then UJS holds.

Proof. Suppose $2 r \leq|y|$. By the change of variable $z=|y| w$, we have

$$
\frac{1}{r^{d}} \int_{B(0, r)} \frac{d z}{|z-y|^{d} \phi(|z-y|)}=\frac{1}{r^{d}} \int_{B\left(0, \frac{r}{|y|}\right)} \frac{d w}{\left|w-\frac{y}{|y|}\right|^{d} \phi(|| y|w-y|)} d w .
$$

Let

$$
A_{y, r}:=\left\{w \in \mathbb{R}^{d}:|w| \leq \frac{r}{|y|},\left|w-\frac{y}{|y|}\right| \leq 1\right\} .
$$

Since $2 r \leq|y|$, it is easy to see that there exists a constant $c_{0}=c_{0}(d)>0$ independent of $r$ and $y$ such that $\mu_{d}\left(A_{y, r}\right) \geq c_{0} r^{d} /|y|^{d}$. Thus, since $\phi$ is increasing,

$$
\frac{1}{r^{d}} \int_{B(0, r)} \frac{d z}{|z-y|^{d} \phi(|z-y|)} \geq \frac{1}{\phi(|y|) r^{d}} \int_{A_{y, r}} \frac{d w}{\left|w-\frac{y}{|y|}\right|^{d}} \geq c_{0} \frac{1}{|y|^{d} \phi(|y|)} .
$$

By the above inequality, we conclude that there exists a constant $c_{1}>0$ such that for every $r>0$ and $2 r \leq|x-y|$, we have

$$
\frac{1}{r^{d}} \int_{B(x, r)} \frac{d z}{|z-y|^{d} \phi(|z-y|)} \geq c_{1} \frac{d z}{|x-y|^{d} \phi(|x-y|)} .
$$

That is, UJS holds when $\kappa_{2}=\kappa_{3}$ in (1.7).

Example 2.2. The examples below clearly satisfy UJS by Lemma 2.1 .

(i) Let $\phi_{1}(r):=\int_{\alpha_{1}}^{\alpha_{2}} r^{\alpha} \nu(d \alpha)$, where $0<\alpha_{1}<\alpha_{2}<2$ and $\nu$ is a probability measure on $\left[\alpha_{1}, \alpha_{2}\right]$. Then it is easy to see that (1.3)-(1.4) are satisfied. Thus if

$$
J(x, y)=\frac{c(x, y) e^{-\gamma|x-y|^{\beta}}}{|x-y|^{d} \int_{\alpha_{1}}^{\alpha_{2}}|x-y|^{\alpha} \nu(d \alpha)},
$$

where $c(x, y)$ is a jointly measurable function that is symmetric in $(x, y)$ and is bounded between two positive constants, then the conditions in Theorem 1.2 hold.

(ii) Similarly, conditions (1.3)-(1.4) are satisfied if $\phi_{1}(r):=\left(\int_{\alpha_{1}}^{\alpha_{2}} r^{-\alpha} \nu(d \alpha)\right)^{-1}$, where $\nu$ is a probability measure on $\left[\alpha_{1}, \alpha_{2}\right] \subset(0,2)$. Thus if

$$
J(x, y)=e^{-\gamma|x-y|^{\beta}} \int_{\alpha_{1}}^{\alpha_{2}} \frac{c(x, y, \alpha)}{|x-y|^{d+\alpha}} \nu(d \alpha),
$$

where $c(\alpha, x, y)$ is a jointly measurable function that is symmetric in $(x, y)$ and is bounded between two positive constants, then the conditions in Theorem 1.2 hold. A particular case occurs when $\nu$ is a discrete measure. Theorem 1.2 in particular, gives the heat kernel estimate for Markov processes on $\mathbb{R}^{d}$ which are linear combinations of independent symmetric temperedstable-like processes (see 23] for tempered stable processes), i.e.,

$$
J(x, y)=e^{-\gamma|x-y|} \sum_{i=1}^{k} \frac{c_{i}(x, y)}{|x-y|^{d+\alpha_{i}}},
$$

where $c_{i}(x, y)$ is a jointly measurable function that is symmetric in $(x, y)$ and is bounded between two positive constants. 
(iii) $\phi_{1}(r)=r^{\alpha(r)}$, where $0 \leq \alpha(r) \leq \beta_{3}<2, \alpha(r) \log r$ is increasing and $(\alpha(2 r)-\alpha(r)) \log r$ is bounded. $\alpha(r)=c-\frac{c}{|r-1|+1}$ is such an example for $c \in(0,2)$. This case corresponds to the jumping density

$$
J(x, y)=\frac{c(x, y) e^{-\gamma|x-y|^{\beta}}}{|x-y|^{d+\alpha(|x-y|)}} .
$$

Example 2.3. (i) Let $Y=\left\{Y_{t}, t \geq 0\right\}$ be the relativistic $\alpha$-stable processes on $\mathbb{R}^{d}$ with mass $m>0$. That is, $\left\{Y_{t}, t \geq 0\right\}$ is a Lévy process on $\mathbb{R}^{d}$ with

$$
\mathbb{E}_{0}\left[\exp \left(i\left\langle\xi, Y_{t}\right\rangle\right)\right]=\exp \left(t\left(m^{\alpha}-\left(|\xi|^{2}+m^{2}\right)^{\alpha / 2}\right)\right),
$$

where $m>0, \alpha \in(0,2)$. It is shown in [14 that the corresponding jumping intensity is

$$
J(x, y)=\frac{\Psi_{\alpha}(m|x-y|)}{|x-y|^{d+\alpha}},
$$

where $\Psi_{\alpha}(r)$ is decreasing and $\Psi_{\alpha}(r)$ is asymptotically equal to $e^{-r}\left(1+r^{(d+\alpha-1) / 2}\right)$ near $r=\infty$, and $\Psi_{\alpha}(r)=1+\Psi_{\alpha}^{\prime \prime}(0) r^{2} / 2+o\left(r^{4}\right)$ near $r=0$. So it is not covered by Example 2.2. However, since $\Psi_{\alpha}(r)$ is decreasing, UJS holds by Lemma 2.1. So the conditions in Theorem 1.2 are satisfied for the jumping intensity kernel for every relativistic $\alpha$-stable process on $\mathbb{R}^{d}$.

When $\alpha=1$, the process is called a relativistic Hamiltonian process. In this case, the heat kernel can be written as

$$
p(t, x, y)=\frac{t}{(2 \pi)^{d} \sqrt{|x-y|^{2}+t^{2}}} \int_{\mathbb{R}^{d}} e^{m t} e^{-\sqrt{\left(|x-y|^{2}+t^{2}\right)\left(|z|^{2}+m^{2}\right)}} d z ;
$$

see [13, Example 2.4]. It can be shown that the estimate of this heat kernel given in [13, page 287, lines 3-5] is the same as that of Theorem 1.2(1) for $\phi(r)=r$ and $\beta=1$.

(ii) Let $Y:=Y^{(1)}+\cdots+Y^{(k)}$, where the $Y^{(i)}$ 's are independent relativistic $\alpha_{i}$-stable processes on $\mathbb{R}^{d}$ with mass $m_{i}$, where $\left\{\alpha_{1}, \cdots, \alpha_{k}\right\} \subset(0,2)$ and $\left\{m_{1}, \cdots, m_{k}\right\} \subset(0, \infty)$. The corresponding jumping intensity is a symmetric kernel given by

$$
J(x, y)=\sum_{i=1}^{k} \frac{\Psi_{\alpha_{i}}\left(m_{i}|x-y|\right)}{|x-y|^{d+\alpha_{i}}} .
$$

Since by (i) each $\frac{\Psi_{\alpha_{i}}\left(m_{i}|x-y|\right)}{|x-y|^{d+\alpha_{i}}}$ satisfies UJS, so does $J$. The other conditions of Theorem 1.2 are also satisfied.

When $\kappa_{2} \neq \kappa_{3}$, conditions (1.1) (1.4) and (1.7) do not need to imply UJS. We give a simple example showing that there is a continuous jumping kernel satisfying conditions (1.1)-(1.4) and (1.7) but not satisfying UJS.

Example 2.4. For convenience, assume $d=1$ and $\alpha \in(0,2)$. Let

$$
U_{n}:=\left\{y:(2 n+1)-e^{-2 n}<|y|<(2 n+1)+e^{-2 n}\right\}, \quad U:=\bigcup_{n=1}^{\infty} U_{n}
$$


and

$$
V_{n}:=\left\{y:(2 n+1)-e^{-2 n-1}<|y|<(2 n+1)+e^{-2 n-1}\right\}, \quad V:=\bigcup_{n=1}^{\infty} V_{n},
$$

and let $\varphi_{0}$ be a continuous function from $(0, \infty)$ to $[1 / 4,1]$ such that

$$
\varphi_{0}(r)= \begin{cases}1 / 4 & \text { on } \bigcup_{n=1}^{\infty}\left\{r:(2 n+1)-e^{-2 n-1}<r<(2 n+1)+e^{-2 n-1}\right\}, \\ 1 & \text { on }(0, \infty) \backslash \bigcup_{n=1}^{\infty}\left\{r:(2 n+1)-e^{-2 n}<r<(2 n+1)+e^{-2 n}\right\} .\end{cases}
$$

Note that $\varphi_{0}$ is not a monotone function. We consider a jumping kernel

$$
J(x, y):=|x-y|^{-1-\alpha} \exp \left(-\varphi_{0}(|x-y|)|x-y|\right),
$$

which is radial and continuous. It is easy to check that the conditions (1.1)-(1.4) and (1.7) hold, but with distinct $\kappa_{2}$ and $\kappa_{3}$. For every $x \in \mathbb{R}^{1}, y \in x+V_{n}$ and $z \in B(x, n), \frac{1}{2}|x-y| \leq|y-z|$. Thus

$$
\begin{aligned}
& \int_{B(x, n)} J(z, y) d y \\
\leq & 2^{1+\alpha}|x-y|^{-1-\alpha} e^{-\frac{1}{4}|x-y|}\left(\int_{B(x, n) \cap(U+y)} e^{\frac{3}{4}|z-y|} d z+\int_{B(x, n) \backslash(U+y)} e^{-\frac{1}{2}|z-y|} d z\right) \\
\leq & 2^{1+\alpha} J(x, y)\left(\int_{B(x, n) \cap(U+y)} e^{\frac{3}{4}|z-y|} d z+\int_{\mathbb{R}^{1}} e^{-\frac{1}{2}|z|} d z\right) \leq c_{1} J(x, y),
\end{aligned}
$$

from which it is easy to show that UJS cannot hold for this $J$.

\section{UPPER BOUND ESTIMATE}

Throughout this section we consider more general non-local Dirichlet forms and their corresponding heat kernels. Set

$$
\mathcal{Q}(f, f)=\int_{\mathbb{R}^{d}} \int_{\mathbb{R}^{d}}(f(y)-f(x))^{2} J_{0}(x, y) d x d y, \quad \mathcal{D}={\overline{C_{c}^{1}\left(\mathbb{R}^{d}\right)}}^{\mathcal{Q}_{1}},
$$

where the jump kernel $J_{0}(x, y)$ is a symmetric non-negative function of $x$ and $y$ so that $\int_{K \times \mathbb{R}^{d}} 1 \wedge|x-y|^{2} J_{0}(x, y) d x d y<\infty$ for every compact subset $K$ of $\mathbb{R}^{d}$. Here $\mathcal{Q}_{1}(f, f):=\mathcal{Q}(f, f)+\|f\|_{2}^{2}, C_{c}^{1}\left(\mathbb{R}^{d}\right)$ denotes the space of $C^{1}$ functions on $\mathbb{R}^{d}$ with compact support, and $\mathcal{D}$ is the closure of $C_{c}^{1}\left(\mathbb{R}^{d}\right)$ with respect to the metric $\mathcal{Q}_{1}(f, f)^{1 / 2}$. The Dirichlet form $(\mathcal{Q}, \mathcal{D})$ is regular on $\mathbb{R}^{d}$, and so it associates a Hunt process $Y$, starting from quasi-everywhere in $\mathbb{R}^{d}$. In the following, the transition density for $Y$ (if it exists) will be denoted by $q(t, x, y)$.

Recall that $\phi$ is the non-decreasing function defined by (1.1) through (1.2)-(1.4).

Proposition 3.1. Suppose there exist positive constants $\kappa_{1}, \kappa_{2}>0$ such that

$$
J_{0}(x, y) \geq \kappa_{1} \frac{1}{|x-y|^{d} \phi(|x-y|)} \quad \text { for all }|y-x| \leq \kappa_{2} .
$$

Then there is a properly $\mathcal{Q}$-exceptional set $\mathcal{N} \subset \mathbb{R}^{d}$, a positive symmetric kernel $q(t, x, y)$ defined on $[0, \infty) \times\left(\mathbb{R}^{d} \backslash \mathcal{N}\right) \times\left(\mathbb{R}^{d} \backslash \mathcal{N}\right)$ and a positive constant $c=$ $c\left(d, \kappa_{1}, \kappa_{2}, \beta, \beta_{1}\right)$ such that

$$
\mathbb{E}_{x}\left[f\left(Y_{t}\right)\right]=\int_{\mathbb{R}^{d}} q(t, x, y) f(y) d y \quad \text { for every } x \in \mathbb{R}^{d} \backslash \mathcal{N} \text { and } t>0,
$$




$$
q(t+s, x, y)=\int_{\mathbb{R}^{d}} q(t, x, z) q(s, z, y) d z \quad \text { for every } t, s>0, x, y \in \mathbb{R}^{d} \backslash \mathcal{N}
$$

and

$$
q(t, x, y) \leq c\left(\phi_{1}^{-1}(t)^{-d} \vee t^{-d / 2}\right) \quad \text { for } t>0 \text { and } x, y \in \mathbb{R}^{d} \backslash \mathcal{N} .
$$

Moreover, there is a $\mathcal{Q}$-nest $\left\{F_{k}, k \geq 1\right\}$ of compact subsets of $\mathbb{R}^{d}$ so that $\mathcal{N}=$ $\mathbb{R}^{d} \backslash \bigcup_{k=1}^{\infty} F_{k}$ and that for every $t>0$ and $y \in \mathbb{R}^{d} \backslash \mathcal{N}, x \mapsto q(t, x, y)$ is continuous on each $F_{k}$.

Proof. For $u \in C_{c}^{1}\left(\mathbb{R}^{d}\right)$, let

$$
\begin{aligned}
\mathcal{E}^{0}(u, u) & :=\kappa_{1} \int_{\mathbb{R}^{d} \times \mathbb{R}^{d}} \frac{(u(x)-u(y))^{2}}{|x-y|^{d} \phi(|x-y|)} \mathbf{1}_{\left\{|x-y| \leq \kappa_{2}\right\}} d x d y, \\
\mathcal{E}^{1}(u, u) & :=\kappa_{1} \int_{\mathbb{R}^{d} \times \mathbb{R}^{d}} \frac{(u(x)-u(y))^{2}}{|x-y|^{d} \phi(|x-y|)} d x d y,
\end{aligned}
$$

and define $\mathcal{D}^{0}:={\overline{C_{c}^{1}\left(\mathbb{R}^{d}\right)}}^{\mathcal{E}_{1}^{0}}, \mathcal{D}^{1}:={\overline{C_{c}^{1}\left(\mathbb{R}^{d}\right)}}^{\mathcal{E}_{1}^{1}}$. Note that under condition (3.2), $\mathcal{Q}(u, u) \geq \mathcal{E}^{0}(u, u)$ for every $u \in C_{c}^{1}\left(\mathbb{R}^{d}\right)$ and hence for every $u \in \mathcal{D}$. Moreover, since

$$
\mathcal{E}^{1}(u, u)-\mathcal{E}^{0}(u, u) \leq 4 \kappa_{1} \int_{\mathbb{R}^{d} \times \mathbb{R}^{d}} \frac{|u(x)|^{2}}{|x-y|^{d} \phi(|x-y|)} \mathbf{1}_{\left\{|x-y|>\kappa_{2}\right\}} d x d y,
$$

it is easy to see that there exists a positive constant $c_{1}>0$ depending on $d, \kappa_{1}, \kappa_{2}$, $\beta, \beta_{1}$ such that

$$
\mathcal{E}_{1}^{1}(u, u) \leq c_{1} \mathcal{E}_{1}^{0}(u, u) \leq c_{1} \mathcal{Q}_{1}(u, u) \quad \text { for every } u \in \mathcal{D} .
$$

Hence $\mathcal{D}^{0}=\mathcal{D}^{1} \supset \mathcal{D}$. Recall that, for $p \geq 1$, the $L^{p}\left(\mathbb{R}^{d}, d x\right)$-norm of a function $u$ on $\mathbb{R}^{d}$ is denoted as $\|u\|_{p}$. It follows from [13, Theorem 3.1] and (3.6) that the following Nash's inequality holds: there is a constant $c_{2}>0$ so that for every $f \in \mathcal{D}$ with $\|f\|_{1}=1$,

$$
\theta\left(\|f\|_{2}\right) \leq c_{2} \mathcal{E}^{1}(f, f) \leq c_{1} c_{2} \mathcal{Q}_{1}(f, f), \quad \text { where } \theta(r)=\frac{r}{\phi\left(r^{-1 / d}\right)} .
$$

Observe that $\left(\mathcal{Q}_{1}, \mathcal{D}\right)$ is the Dirichlet form of the 1-subprocess of $Y$. We have by [1, Theorem 3.1] and the same way as that for [13, Theorem 3.2] using (3.7), that there is a properly $\mathcal{Q}$-exceptional set $\mathcal{N} \subset \mathbb{R}^{d}$ and a positive symmetric kernel $q(t, x, y)$ defined on $[0, \infty) \times\left(\mathbb{R}^{d} \backslash \mathcal{N}\right) \times\left(\mathbb{R}^{d} \backslash \mathcal{N}\right)$ such that for every $x \in \mathbb{R}^{d} \backslash \mathcal{N}$ and $t>0$, (3.3) -3.4) are true, and that for every $x, y \in \mathbb{R}^{d} \backslash \mathcal{N}$,

$$
q(t, x, y) \leq c_{3} e^{t} \phi^{-1}(t)^{-d} \quad \text { for } t>0 .
$$

Moreover, there is a $\mathcal{Q}$-nest $\left\{F_{k}, k \geq 1\right\}$ of compact subsets of $\mathbb{R}^{d}$ so that $\mathcal{N}=$ $\mathbb{R}^{d} \backslash \bigcup_{k=1}^{\infty} F_{k}$ and that for every $t>0$ and $y \in \mathbb{R}^{d} \backslash \mathcal{N}, x \mapsto q(t, x, y)$ is continuous on each $F_{k}$.

On the other hand, by the third line from the end of the proof of 10, Proposition $2.2]$, there is a constant $c_{4}>0$ so that for every $u \in \mathcal{D} \subset W^{\beta_{1} / 2,2}\left(\mathbb{R}^{d}\right)$ with 
$\mathcal{Q}(u, u) \leq\|u\|_{1}^{2}$,

$$
\|u\|_{2}^{2} \leq c_{4} \mathcal{Q}(u, u)^{\frac{d}{d+2}}\|u\|_{1}^{\frac{4}{d+2}} .
$$

Therefore by [6, Theorem 2.9] and [1, Theorem 3.1], we have

$$
q(t, x, y) \leq c_{5} t^{-d / 2} \quad \text { for every } t \geq 1 \text { and } x, y \in \mathbb{R}^{d} \backslash \mathcal{N} .
$$

Combining this, (3.8) and the observation that $e^{t} \phi^{-1}(t)^{-d} \leq e \phi_{1}^{-1}(t)^{-d}$ for $t \in$ $(0,1]$ and the fact that $\phi_{1}^{-1}(t)^{-d} \geq c_{6} t^{-d / 2}$ for $t \in(0,1]$ and $\phi_{1}^{-1}(t)^{-d} \leq c_{7} t^{-d / 2}$ for $t \geq 1$, we conclude that (3.5) holds.

Let $\psi_{2}$ be an increasing function on $[0, \infty)$ with $\psi_{2}(r)=1$ for $0<r \leq 1$ and

$$
c_{1} e^{\gamma_{3} r^{\beta_{0}}} \leq \psi_{2}(r) \leq c_{2} e^{\gamma_{4} r^{\beta_{0}}} \quad \text { for every } 1<r<\infty
$$

for some constants $\gamma_{4} \geq \gamma_{3}>0$. Here $\beta_{0} \in(0, \beta]$, where $\beta$ is the constant given in (1.2). We also let $\phi_{2}$ be a strictly increasing function on $[0, \infty)$ with $\phi_{2}(0)=0$, $\phi_{2}(1)=1$,

$$
c_{1}\left(\frac{R}{r}\right)^{\beta_{3}} \leq \frac{\phi_{2}(R)}{\phi_{2}(r)} \leq c_{2}\left(\frac{R}{r}\right)^{\beta_{4}} \quad \text { for every } 0<r<R<\infty
$$

and

$$
\int_{0}^{r} \frac{s}{\phi_{2}(s)} d s \leq c_{3} \frac{r^{2}}{\phi_{2}(r)} \quad \text { for every } r>0
$$

for some $0<c_{1}<c_{2}, c_{3}>0$ and $0<\beta_{3} \leq \beta_{4}<2$. (Note that $\phi_{2}, \psi_{2}$ can be different from $\phi_{1}, \psi_{1}$, which were given in (1.1).)

Now we further assume that there exists a positive constant $\kappa_{1}>0$ such that for every $x, y \in \mathbb{R}^{d}$,

$$
\begin{aligned}
& \kappa_{1}^{-1} \frac{1}{|x-y|^{d} \phi_{1}(|x-y|) \psi_{1}(|x-y|)} \\
& \leq J_{0}(x, y) \leq \kappa_{1} \frac{1}{|x-y|^{d} \phi_{2}(|x-y|) \psi_{2}(|x-y|)} .
\end{aligned}
$$

In the remainder of this section we will use the following Davies' method many times: using Proposition 3.1, (1.3), [1, Theorems 3.1 and 3.2], and [6, Corollary 3.28], we have that for every $x, y \in \mathbb{R}^{d} \backslash \mathcal{N}$ and $t>0$,

$$
q(t, x, y) \leq c_{1}\left(t^{-d / \beta_{1}} \vee t^{-d / 2}\right) \exp (-E(2 t, x, y)) .
$$

Here $E(2 t, x, y)$ is given by the following:

$$
\begin{aligned}
\Gamma(f)(x) & :=\int\left(e^{f(x)-f(y)}-1\right)^{2} J_{0}(x, y) d y, \Lambda(f)^{2}:=\|\Gamma(f)\|_{\infty} \vee\|\Gamma(-f)\|_{\infty}, \\
E(t, x, y) & :=\sup \left\{|f(x)-f(y)|-t \Lambda(f)^{2}: f \in \operatorname{Lip}_{c} \text { with } \Lambda(f)<\infty\right\},
\end{aligned}
$$

where $\operatorname{Lip}_{c}$ is a space of compactly supported Lipschitz continuous functions on $\mathbb{R}^{d}$.

Theorem 3.2. Suppose the jumping kernel $J_{0}$ satisfies (3.12) and $\beta_{0} \geq 1$. Then for every $0<C_{*}<1$ there exist $c_{1}, c_{2}>0$ such that

$$
q(t, x, y) \leq c_{1}\left(t^{-d / \beta_{1}} \vee t^{-d / 2}\right) \exp \left(-\frac{c_{2}|x-y|^{2}}{t}\right)
$$


for $x, y \in \mathbb{R}^{d} \backslash \mathcal{N}$ and $t>0$ with $|x-y| \leq t / C_{*}$, and

$$
q(t, x, y) \leq c_{1}\left(t^{-d / \beta_{1}} \vee t^{-d / 2}\right) \exp \left(-c_{2}|x-y|\left(\log \frac{|x-y|}{t}\right)^{\frac{\beta_{0}-1}{\beta_{0}}}\right)
$$

for $x, y \in \mathbb{R}^{d} \backslash \mathcal{N}$ and $t>0$ with $|x-y|>t / C_{*}$.

Proof. Fix $x, y \in \mathbb{R}^{d}$ and let $r=|x-y|$. Define $f(\xi):=\lambda(|\xi-x| \wedge r)$ for $\xi \in \mathbb{R}^{d}$, where $\lambda$ is a constant to be chosen later. Note that $|f(\xi)-f(\eta)| \leq \lambda|\xi-\eta|$ for every $\xi, \eta \in \mathbb{R}^{d}$. Since $\left|e^{s}-1\right|^{2} \leq s^{2} e^{2|s|}$,

$$
\begin{aligned}
\Gamma(f)(\xi) & =\int_{\mathbb{R}^{d}}\left(e^{f(\xi)-f(\eta)}-1\right)^{2} J_{0}(\xi, \eta) d \eta \leq \lambda^{2} \int_{\mathbb{R}^{d}}|\xi-\eta|^{2} e^{2 \lambda|\xi-\eta|} J_{0}(\xi, \eta) d \eta \\
& \leq \kappa_{1} \lambda^{2} \int_{\mathbb{R}^{d}} \frac{e^{2 \lambda|\xi-\eta|-\gamma_{3}|\xi-\eta|^{\beta_{0}}}}{|\xi-\eta|^{d-2} \phi_{2}(|\xi-\eta|)} d \eta \leq c_{1} \lambda^{2} \int_{0}^{\infty} \frac{s e^{2 \lambda s-\gamma_{3} s^{\beta_{0}}}}{\phi_{2}(s)} d s,
\end{aligned}
$$

where the lower bound of (3.9) is used in the second-to-last inequality.

We first prove (3.14). When $0<\lambda \leq \gamma_{3} / 4$,

$$
\Gamma(f)(\xi) \leq c_{2} \lambda^{2}\left(\int_{0}^{1} \frac{s}{\phi_{2}(s)} d s+\frac{1}{\phi_{2}(1)} \int_{1}^{\infty} s e^{-\gamma_{3} s / 2} d s\right) \leq c_{3} \lambda^{2},
$$

where we used (3.11) in the last inequality. So we have

$$
-E(2 t, x, y) \leq-\lambda r+c_{3} t \lambda^{2}=\lambda\left(-r+c_{3} t \lambda\right) \quad \text { for all } \lambda \leq \gamma_{3} / 4
$$

Choose $c_{3}$ larger if necessary so that $c_{3} \geq \frac{2}{C_{*} \gamma_{3}}$. For each $r \leq t / C_{*}$, take

$$
\lambda:=\frac{r}{2 c_{3} t} \leq \frac{1}{2 c_{3} C_{*}} \leq \frac{\gamma_{3}}{4} .
$$

Then from (3.17) we get $-E(2 t, x, y) \leq-\frac{r^{2}}{4 c_{3} t}$. Putting this into (3.13), we obtain (3.14) for $|\xi-\eta| \leq t / C_{*}$.

We next prove (3.15). With $c_{4}:=\left(4 / \gamma_{3}\right)^{1 /\left(\beta_{0}-1\right)}$, we have by (3.16),

$$
\begin{aligned}
\Gamma(f)(\xi) & \leq c_{2} \lambda^{2} \int_{0}^{c_{4} \lambda^{1 /\left(\beta_{0}-1\right)}} \frac{s e^{2 \lambda s}}{\phi_{2}(s)} d s+c_{2} \lambda^{2} \int_{c_{4} \lambda^{1 /\left(\beta_{0}-1\right)}}^{\infty} \frac{s e^{-\gamma_{3} s^{\beta_{0} / 2}}}{\phi_{2}(s)} d s \\
& \leq c_{5} \lambda^{2}\left(\frac{\left(c_{4} \lambda^{1 /\left(\beta_{0}-1\right)}\right)^{2}}{\phi_{2}\left(c_{4} \lambda^{1 /\left(\beta_{0}-1\right)}\right)} e^{2 c_{4} \lambda^{\beta_{0} /\left(\beta_{0}-1\right)}}+1\right) \leq c_{6} \lambda^{2} e^{c_{7} \lambda^{\beta_{0} /\left(\beta_{0}-1\right)}},
\end{aligned}
$$

where we used (3.11) and (3.10) in the second and last inequality, respectively. So we have that for every $\lambda>0$,

$$
-E(2 t, x, y) \leq-\lambda r+c_{6} t \lambda^{2} e^{c_{7} \lambda^{\beta_{0} /\left(\beta_{0}-1\right)}}=\lambda r\left(c_{6} \lambda(t / r) e^{c_{7} \lambda^{\beta_{0} /\left(\beta_{0}-1\right)}}-1\right) .
$$

Choose $c_{8} \leq\left(2 c_{7}\right)^{-\left(\beta_{0}-1\right) / \beta_{0}}$ small so that

$$
c_{6} c_{8}\left(\sup _{a \geq C_{*}^{-1}}(\log a)^{\left(\beta_{0}-1\right) / \beta_{0}} a^{-1 / 2}\left(C_{*}^{1 / 2} \vee 1\right)\right)<\frac{1}{2}
$$

and take $\lambda:=c_{8}(\log (r / t))^{\left(\beta_{0}-1\right) / \beta_{0}}$. Then

$$
\begin{aligned}
(t / r) e^{c_{7} \lambda^{\beta_{0} /\left(\beta_{0}-1\right)}} & =(t / r)(r / t)^{c_{7} c_{8}^{\beta_{0} /\left(\beta_{0}-1\right)}} \leq(t / r)\left(C_{*}^{1 / 2} \vee 1\right)(r / t)^{1 / 2} \\
& =\left(C_{*}^{1 / 2} \vee 1\right)(r / t)^{-1 / 2}
\end{aligned}
$$


Thus from (3.18) we get

$$
\begin{aligned}
-E(2 t, x, y) & \leq \lambda r\left(c_{6} c_{8}(\log (r / t))^{\left(\beta_{0}-1\right) / \beta_{0}}\left(C_{*}^{1 / 2} \vee 1\right)(r / t)^{-1 / 2}-1\right) \\
& \leq-\frac{1}{2} \lambda r=-\frac{1}{2} c_{8} r(\log (r / t))^{\left(\beta_{0}-1\right) / \beta_{0}} .
\end{aligned}
$$

Putting this into (3.13), we obtain (3.15) for $|x-y|>t / C_{*}$.

We will use the following Meyer's construction 22 several times in this section. Let $\lambda>0$ and define

$$
J^{(\lambda)}(\xi, \eta):=\mathbf{1}_{\{|\xi-\eta| \leq \lambda\}} J_{0}(\xi, \eta) \text { and } J_{\lambda}(\xi, \eta):=\mathbf{1}_{\{|\xi-\eta|>\lambda\}} J_{0}(\xi, \eta) .
$$

One can remove the jumps of $Y$ of size larger than $\lambda$ to obtain a new process $Y^{(\lambda)}$ as follows. One starts with the process $Y$ associated with jumping kernel $J_{0}$, runs it until the stopping time $T_{1}:=\inf \left\{t:\left|Y_{t-}-Y_{t}\right|>\lambda\right\}$, and at that time restarts $Y$ at the point $Y_{T_{1}-}$. One then repeats this procedure. Meyer [22] showed that the resulting process $Y^{(\lambda)}$ is a process with jumping kernel $J^{(\lambda)}$. From this construction we see that $Y^{(\lambda)}$ can start from every point in $\mathbb{R}^{d} \backslash \mathcal{N}$ and that it admits quasicontinuous transition density function $q^{(\lambda)}(t, x, y)$ defined on $(0, \infty) \times\left(\mathbb{R}^{d} \backslash \mathcal{N}\right) \times$ $\left(\mathbb{R}^{d} \backslash \mathcal{N}\right)$.

It is easy to see that the Dirichlet form of $Y^{(\lambda)}$ on $L^{2}\left(\mathbb{R}^{d}, d x\right)$ is $\left(\mathcal{Q}^{(\lambda)}, \mathcal{D}\right)$, where

$$
\mathcal{Q}^{(\lambda)}(v, v)=\int_{\mathbb{R}^{d}} \int_{\mathbb{R}^{d}}(v(\xi)-v(\eta))^{2} J^{(\lambda)}(\xi, \eta) d \eta d \xi .
$$

In fact, note that by (3.10), $\int_{\mathbb{R}^{d}} J_{\lambda}(\eta, \xi) d \xi \leq c_{1}(\lambda)<\infty$. Thus we have for $v \in \mathcal{D}$,

$$
0 \leq \mathcal{Q}(v, v)-\mathcal{Q}^{(\lambda)}(v, v) \leq 4 \int_{\mathbb{R}^{d}} v(\xi)^{2}\left(\int_{\mathbb{R}^{d}} J_{\lambda}(\xi, \eta) d \eta\right) d \xi \leq 4 c_{1}(\lambda) \int_{\mathbb{R}^{d}} v(\xi)^{2} d \xi,
$$

and so

$$
\left(1+4 c_{1}(\lambda)\right)^{-1} \mathcal{Q}_{1}(v, v) \leq \mathcal{Q}_{1}^{(\lambda)}(v, v) \leq \mathcal{Q}_{1}(v, v) \quad \text { for every } v \in \mathcal{D}
$$

By Proposition 3.1, for each $b>0$ there exists a constant $c=c(b, d)>0$ such that for every $\lambda \geq b$,

$$
q^{(\lambda)}(t, x, y) \leq c\left(\left(\phi_{1}^{-1}(t)\right)^{-d} \vee t^{-d / 2}\right) \quad \forall t \in(0, \infty) \text { and } x, y \in \mathbb{R}^{d} \backslash \mathcal{N} .
$$

Theorem 3.3. Suppose the jumping kernel satisfies (3.12) and with $\beta_{0} \leq 1$. Then for every $a, b>0$, there exist $c_{k}, k=1, \cdots, 4$, and $C_{*} \in(0,1]$ such that for every $t \geq a$ and $x, y \in \mathbb{R}^{d} \backslash \mathcal{N}$ with $|x-y| \geq b$,

$$
q(t, x, y) \leq c_{1} \begin{cases}t^{-d / 2} e^{-c_{2}|x-y|^{2} / t} & \text { when } t>C_{*}|x-y|^{2-\beta_{0}} \\ e^{-c_{2}|x-y|^{\beta_{0}}} & \text { when } t \leq C_{*}|x-y|^{2-\beta_{0}}\end{cases}
$$

or, equivalently, $q(t, x, y) \leq c_{3} t^{-d / 2} e^{-c_{4}\left(\frac{|x-y|^{2}}{t} \wedge|x-y|^{\beta_{0}}\right)}$.

Proof. For $\lambda>0$ and the function $f$ on $\mathbb{R}^{d}$, define

$$
\begin{aligned}
\Gamma_{\lambda}(f)(\xi) & :=\int_{\mathbb{R}^{d}}\left(e^{f(\xi)-f(\eta)}-1\right)^{2} J^{(\lambda)}(\xi, \eta) d \eta \\
\text { and } \quad \Lambda_{\lambda}(f)^{2} & :=\left\|\Gamma_{\lambda}(f)\right\|_{\infty} \vee\left\|\Gamma_{\lambda}(-f)\right\|_{\infty}
\end{aligned}
$$


For fixed $x, y \in \mathbb{R}^{d} \backslash \mathcal{N}$, let $f(\xi):=s(|\xi-x| \wedge|x-y|)$ for $\xi \in \mathbb{R}^{d}$, where $s>0$ is a constant to be chosen later. Note that $|f(\eta)-f(\xi)| \leq s|\xi-\eta|$. We have by the same argument as that for (3.16) that

$$
\Gamma_{\lambda}(f)(\xi) \leq c_{1} s^{2} \int_{0}^{\lambda} \frac{u}{\phi_{2}(u)} e^{2 s u-\gamma_{3} u^{\beta_{0}}} d u \quad \text { for every } \xi \in \mathbb{R}^{d},
$$

where $\gamma_{3}>0$ is the constant in (3.9). With $s=\gamma_{3} \lambda^{\beta_{0}-1} / 4$, we have that for all $u \leq \lambda$,

$$
2 s u-\gamma_{3} u^{\beta_{0}}=\gamma_{3} \lambda^{\beta_{0}-1} u / 2-\gamma_{3} u^{\beta_{0}}=\gamma_{3} u^{\beta_{0}}\left((u / \lambda)^{1-\beta_{0}} / 2-1\right) \leq-\gamma_{3} u^{\beta_{0}} / 2,
$$

which implies that

$$
\Gamma_{\lambda}(f)(\xi) \leq C s^{2}, \quad \text { where } C:=c_{1} \int_{0}^{\infty} \frac{u}{\phi_{2}(u)} e^{-\gamma_{3} u^{\beta_{0}} / 2} d u
$$

(Note that $C<\infty$ due to (3.11) and $\beta_{0}>0$.) The same estimate holds for $\Gamma_{\lambda}(-f)(\xi)$. Thus, with $\lambda:=|x-y|$,

$$
-|f(y)-f(x)|+\Lambda_{\lambda}(f)^{2} t \leq s(-\lambda+C t s)=\frac{\gamma_{3}}{4} \lambda^{\beta_{0}-1}\left(-\lambda+\frac{C \gamma_{3}}{4}\left(\frac{t}{\lambda^{2-\beta_{0}}}\right) \lambda\right) .
$$

Set $C_{*}:=2 /\left(C \gamma_{3}\right)$. Then

$$
-|f(y)-f(x)|+\Lambda_{\lambda}(f)^{2} t \leq-\frac{\gamma_{3}}{8} \lambda^{\beta_{0}} \quad \text { if } 0<t \leq C_{*} \lambda^{2-\beta_{0}} .
$$

On the other hand, if $t \geq C_{*} \lambda^{2-\beta_{0}}$, with $s:=\frac{\lambda}{2 C t}=\frac{\gamma_{3} C_{*} \lambda}{4 t} \leq \frac{\gamma_{3}}{4} \lambda^{\beta_{0}-1}$, we have that for all $u \leq \lambda$,

$$
2 s u-\gamma_{3} u^{\beta_{0}} \leq \frac{\gamma_{3}}{2} \lambda^{\beta_{0}-1} u-\gamma_{3} u^{\beta_{0}}=-\gamma_{3} u^{\beta_{0}}\left(-\frac{1}{2}(u / \lambda)^{1-\beta_{0}}+1\right) \leq-\gamma_{3} u^{\beta_{0}} / 2 .
$$

Therefore, we have by (3.22) that for $t>C_{*} \lambda^{2-\beta_{0}}$,

(3.24) $-|f(y)-f(x)|+\Lambda_{\lambda}(f)^{2} t \leq s(-\lambda+C t s)=\frac{\lambda}{2 C t}\left(-\lambda+\frac{\lambda}{2}\right)=-\frac{1}{4 C} \frac{\lambda^{2}}{t}$.

By [6, Corollary 3.28], [1, Theorems 3.1 and 3.2] and (3.19), there exists a constant $c_{2}=c_{2}(b)>0$, independent of $\lambda \geq b$ such that

$$
q^{(\lambda)}(t, x, y) \leq c_{2}\left(t^{-d / \beta_{1}} \vee t^{-d / 2}\right) \exp \left(-|f(y)-f(x)|+\Lambda_{\lambda}(f)^{2} t\right) .
$$

Thus applying our (3.23)-(3.24) with $\lambda=|x-y|$, there exists a constant $c_{3}>0$ such that for every $|x-y| \geq b$,

$$
\begin{aligned}
& q^{(\lambda)}(t, x, y) \\
\leq & c_{2}\left(t^{-d / \beta_{1}} \vee t^{-d / 2}\right) \begin{cases}e^{-c_{3}|x-y|^{2} / t} & \text { when } C_{*}|x-y|^{2-\beta_{0}}<t, \\
e^{-c_{3}|x-y|^{\beta_{0}}} & \text { when } C_{*}|x-y|^{2-\beta_{0}} \geq t .\end{cases}
\end{aligned}
$$

Note that by (3.9) and (3.10) there are constants $c_{4}, c_{5}>0$ such that for every $\lambda>0$,

$$
\left\|J_{\lambda}\right\|_{\infty} \leq \frac{c_{4}}{\lambda^{d+\beta_{3}}} e^{-\gamma_{3} c_{5} \lambda^{\beta_{0}}}
$$


It then follows from (3.26) and [3] Lemma 3.2] that for every $t>0$ and $|x-y| \geq b$, we have by taking $\lambda=|x-y|$ that

$$
\begin{aligned}
& q(t, x, y) \leq q^{(\lambda)}(t, x, y)+t \sup _{\xi \in \mathbb{R}^{d}}\left\|J_{\lambda}(\xi, \cdot)\right\|_{\infty} \\
& \leq c_{6}\left\{\begin{array}{l}
\left(t^{-d / \beta_{1}} \vee t^{-d / 2}\right) e^{-c_{3}|x-y|^{2} / t}+\frac{t}{|x-y|^{d+\beta_{3}}} e^{-c_{7}|x-y|^{\beta_{0}}} \\
\left(t^{-d / \beta_{1}} \vee t^{-d / 2}\right) e^{-c_{3}|x-y|^{\beta_{0}}}+\frac{t}{\text { when } C_{*}|x-y|^{2-\beta_{0}}}<t, \\
\begin{array}{c}
|x-y|^{d+\beta_{3}} \\
\text { when } C_{*}|x-y|^{2-\beta_{0}} \geq t .
\end{array}
\end{array}\right.
\end{aligned}
$$

Therefore, for every $t \geq a>0$ and $|x-y| \geq b>0$,

$$
q(t, x, y) \leq c_{8} \begin{cases}t^{-d / 2} e^{-c_{9}|x-y|^{2} / t} & \text { when } C_{*}|x-y|^{2-\beta_{0}}<t<|x-y|^{2}, \\ e^{-c_{9}|x-y|^{\beta_{0}}} & \text { when } C_{*}|x-y|^{2-\beta_{0}} \geq t .\end{cases}
$$

When $t \geq a>0$ and $t \geq|x-y|^{2}$, we have by Proposition 3.1 that

$$
q(t, x, y) \leq c_{10} t^{-d / 2} \leq c_{11} t^{-d / 2} e^{-c_{9}|x-y|^{2} / t} .
$$

This completes the proof of the theorem.

The estimates for the following short time region require more sophisticated choices of test functions in order to obtain the right polynomial exponents.

Theorem 3.4. Suppose the jumping kernel satisfies (3.12) and $\beta_{0}>0$. Then for every $a>0$ there exist $c_{1}, c_{2}>0$ and $C^{*} \in(0,1]$ such that for every $0<t \leq a$ and $x, y \in \mathbb{R}^{d} \backslash \mathcal{N}$ with $|x-y| \geq a / C^{*}$,

$$
q(t, x, y) \leq c_{1} t e^{-c_{2}|x-y|^{\beta_{0} \wedge 1}} .
$$

Suppose further that $\beta_{0} \geq 1$. Then, for every $0<t \leq a$ and $|x-y| \geq a / C^{*}$,

$$
\begin{aligned}
& q(t, x, y) \\
\leq & c_{3} t\left(\exp \left(-c_{2}|x-y|(\log (|x-y| / t))^{\left(\beta_{0}-1\right) / \beta_{0}}\right)+\exp \left(-c_{2}|x-y|^{\beta_{0}}\right)\right) .
\end{aligned}
$$

Proof. Fix $x, y \in \mathbb{R}^{d} \backslash \mathcal{N}$ with $|x-y| \geq a / C^{*}$, where $C^{*}>0$ is a constant to be chosen later. Let $\lambda \geq \frac{a \beta_{3}}{d+\beta_{3}}$ be a constant to be chosen later. Recall the definition of $\Gamma_{\lambda}(f)$ and $\Lambda_{\lambda}(f)^{2}$ from (3.20). Let $r:=|x-y|$ and

$$
f(\xi):=\frac{s+g}{3}(|\xi-x| \wedge r) \quad \text { for } \xi \in \mathbb{R}^{d},
$$

where $s$ and $g$ are two positive constants to be chosen later. Since $|f(\eta)-f(\xi)| \leq$ $\frac{s+g}{3}|\xi-\eta|$ for all $\xi, \eta \in \mathbb{R}^{d}$, by the same argument as that for (3.16) we have that

$$
\Gamma_{\lambda}(f)(\xi) \leq \frac{C}{3}(s+g)^{2} e^{2 s \lambda / 3} \int_{0}^{\lambda} \frac{u e^{2 g u / 3-\gamma_{3} u^{\beta_{0}}}}{\phi_{2}(u)} d u .
$$

We first establish (3.27). Taking $g=\gamma_{3} \lambda^{\left(\beta_{0}-1\right) \wedge 0}$, we have by (3.29) that

$$
\begin{aligned}
\Gamma_{\lambda}(f)(\xi) & \leq \frac{C}{3}\left(s+\gamma_{3} \lambda^{\left(\beta_{0}-1\right) \wedge 0}\right)^{2} e^{2 s \lambda / 3}\left(c_{1}+c_{2} \int_{1}^{\lambda \vee 1} u e^{-c_{3} \gamma_{3} u^{\beta_{0}} / 3} d u\right) \\
& \leq \frac{C_{1}}{3}\left(s+\gamma_{3} \lambda^{\left(\beta_{0}-1\right) \wedge 0}\right)^{2} e^{2 s \lambda / 3}
\end{aligned}
$$


for every $\xi \in \mathbb{R}^{d}$, where the lower bound of (3.10) is used in the first inequality. The constant $C_{1}>0$ is independent of $\lambda>0$. The same estimate holds for $\Gamma_{\lambda}(-f)(\xi)$. Thus,

$$
\begin{aligned}
& -|f(y)-f(x)|+\Lambda_{\lambda}(f)^{2} t \\
\leq & \frac{s+\gamma_{3} \lambda^{\left(\beta_{0}-1\right) \wedge 0}}{3} r\left(-1+C_{1}\left(s+\gamma_{3} \lambda^{\left(\beta_{0}-1\right) \wedge 0}\right)(t / r) e^{2 s \lambda / 3}\right) .
\end{aligned}
$$

Now take $s=\frac{6}{r \beta_{3}}\left(d+\beta_{3}\right) \log \left(\frac{r}{t}\right)$. Then for $\lambda=\frac{\beta_{3}}{8\left(d+\beta_{3}\right)} r$ we have $(t / r) e^{2 s \lambda / 3}=$ $(t / r) e^{\frac{1}{2} \log \frac{r}{t}}=\sqrt{t / r}$. Choose $C^{*} \in(0,1]$ such that

$$
\frac{6\left(d+\beta_{3}\right)}{\beta_{3}} C_{1}\left(a^{-1} C^{*}\left(\sup _{0<v \leq C^{*}} \sqrt{v} \log \frac{1}{v}\right)+\gamma_{3}\left(\frac{a \beta_{8}}{8\left(d+\beta_{3}\right)}\right)^{\left(\beta_{0}-1\right) \wedge 0}\left(\sup _{0<v \leq C^{*}} \sqrt{v}\right)\right)
$$

is less than $1 / 2$, where $C_{1}>0$ is the constant in (3.30). Then for $0<t \leq a$, $r \geq a / C^{*}$ and $\lambda=\frac{\beta_{3}}{8\left(d+\beta_{3}\right)} r$, we have by (3.30),

$$
\begin{aligned}
(3.31) & -|f(y)-f(x)|+\Lambda_{\lambda}(f)^{2} t \\
\leq & \frac{s+\gamma_{3} \lambda^{\left(\beta_{0}-1\right) \wedge 0}}{3} r \\
& \times\left(-1+\frac{6\left(d+\beta_{3}\right)}{\beta_{3}} a^{-1} C^{*} C_{1} \sqrt{\frac{t}{r}} \log \left(\frac{r}{t}\right)+C_{1} \gamma_{3}\left(\frac{a \beta_{3}}{8\left(d+\beta_{3}\right)}\right)^{\left(\beta_{0}-1\right) \wedge 0} \sqrt{\frac{t}{r}}\right) \\
\leq & -\frac{s+\gamma_{3} \lambda^{\left(\beta_{0}-1\right) \wedge 0}}{6} r \\
\leq & -\left(\frac{d}{\beta_{3}}+1\right) \log \frac{r}{t}-\frac{\gamma_{3}}{6}\left(\frac{\beta_{3}}{8\left(d+\beta_{3}\right)}\right)^{\left(\beta_{0}-1\right) \wedge 0} r^{\left(\beta_{0} \wedge 1\right) .}
\end{aligned}
$$

Let $b:=\frac{a \beta_{3}}{8\left(d+\beta_{3}\right) C^{*}}$ and note that $\phi_{1}^{-1}(t) \leq c_{1} \phi_{2}^{-1}(t)$ for $t \leq 1$ due to (3.12). By (3.10) and (3.25) there exists a constant $c_{1}>0$, independent of $\lambda \geq b$, such that

$$
q^{(\lambda)}(t, x, y) \leq c_{1} t^{-d / \beta_{3}} \exp \left(-|f(y)-f(x)|+\Lambda_{\lambda}(f)^{2} t\right)
$$

for all $t \in(0, a], x, y \in \mathbb{R}^{d} \backslash \mathcal{N}$. Thus by (3.31), for $x, y \in \mathbb{R}^{d} \backslash \mathcal{N}$ with $|x-y| \geq a / C^{*}$ and $t \in(0, a]$, we have by taking $\lambda:=\frac{\beta_{3}}{8\left(d+\beta_{3}\right)}|x-y| \geq b$ that

$$
\begin{aligned}
& q^{(\lambda)}(t, x, y) \\
\leq & c_{1} t^{-d / \beta_{3}}\left(\frac{t}{C^{*}|x-y|}\right)^{\frac{d}{\beta_{3}}+1} e^{-\gamma_{3} c_{2}|x-y|^{\beta_{0} \wedge 1} / 6} \leq c_{3} t e^{-\gamma_{3} c_{2}|x-y|^{\beta_{0} \wedge 1} / 6} .
\end{aligned}
$$

Note that by (3.9) and (3.10) there are constants $c_{4}, c_{5}>0$ such that for every $\lambda \geq b$,

$$
\left\|J_{\lambda}\right\|_{\infty} \leq \frac{c_{4}}{\lambda^{d+\beta_{3}}} e^{-\gamma_{3} c_{5} \lambda^{\beta_{0} \wedge 1}} \leq c_{4} b^{-\left(d+\beta_{3}\right)} e^{-\gamma_{3} c_{5} \lambda^{\beta_{0} \wedge 1}} .
$$

It then follows from (3.33) and [3, Lemma 3.2] that

$$
q(t, x, y) \leq q^{(\lambda)}(t, x, y)+t \sup _{\xi \in \mathbb{R}^{d}}\left\|J_{\lambda}(\xi, \cdot)\right\|_{\infty} \leq c_{6} t e^{-\gamma_{3} c_{7}|x-y|^{\beta_{0} \wedge 1}} .
$$

This proves (3.27). 
We next show that (3.28) holds. Let $c_{+}:=\left(2 / \gamma_{3}\right)^{1 /\left(\beta_{0}-1\right)}$. Then we have by (3.29) that for every $\xi \in \mathbb{R}^{d}$,

$$
\begin{aligned}
& \Gamma_{\lambda}(f)(\xi) \\
\leq & \frac{C}{3}(s+g)^{2} e^{2 s \lambda / 3}\left(\int_{0}^{c_{+} g^{1 /\left(\beta_{0}-1\right)}} \frac{u}{\phi_{2}(u)} e^{g u} d u+c_{2} \int_{c_{+} g^{1 /\left(\beta_{0}-1\right)}}^{\infty} \frac{u}{\phi_{2}(u)} e^{-\gamma_{3} u^{\beta_{0}} / 2} d u\right) \\
\leq & \frac{C}{3}(s+g)^{2} e^{2 s \lambda / 3}\left(\frac{\left(c_{+} g^{1 /\left(\beta_{0}-1\right)}\right)^{2}}{\phi_{2}\left(c_{+} g^{1 /\left(\beta_{0}-1\right)}\right)} e^{c_{+} g^{\beta_{0} /\left(\beta_{0}-1\right)}}+c_{3}\right) \\
\leq & \frac{C_{2}}{3}(s+g)^{2} e^{2 s \lambda / 3} e^{g^{\beta_{0} /\left(\beta_{0}-1\right)} /\left(4 C_{3}\right)},
\end{aligned}
$$

where $C_{2}$ and $C_{3}$ are positive constants that are independent of $g>0$. In the second-to-last inequality, (3.11) is used. The same estimate holds for $\Gamma_{\lambda}(-f)(\xi)$. Now take $g:=C_{3}\left(\log \frac{r}{t}\right)^{\left(\beta_{0}-1\right) / \beta_{0}}$. Then for $t>0$,

$$
\begin{aligned}
& -|f(y)-f(x)|+\Lambda_{\lambda}(f)^{2} t \\
\leq & \frac{s+g}{3}\left(-r+C_{2}\left(s+C_{3}\left(\log \frac{r}{t}\right)^{\left(\beta_{0}-1\right) / \beta_{0}}\right) e^{2 s \lambda / 3}\left(\frac{r}{t}\right)^{1 / 4} t\right) \\
= & \frac{s+g}{3}\left(r\left(-1+C_{2}\left(s+C_{3}\left(\log \frac{r}{t}\right)^{\left(\beta_{0}-1\right) / \beta_{0}}\right) e^{2 s \lambda / 3}\left(\frac{t}{r}\right)^{3 / 4}\right)\right) .
\end{aligned}
$$

Next we take $s:=\frac{6}{r \beta_{3}}\left(d+\beta_{3}\right) \log \left(\frac{r}{t}\right)$. Then for $\lambda=\frac{\beta_{3}}{8\left(d+\beta_{3}\right)} r$,

$$
e^{2 s \lambda / 3}\left(\frac{t}{r}\right)^{3 / 4}=\exp \left(\frac{1}{2}\left(\log \frac{r}{t}\right)\right)\left(\frac{t}{r}\right)^{3 / 4}=\left(\frac{t}{r}\right)^{\frac{1}{4}} .
$$

Choose $C_{*} \in\left(0, \frac{1}{2}\right]$ such that

$$
C_{2} \sup _{0<v<C_{*}}\left(\frac{6}{a \beta_{3}}\left(d+\beta_{3}\right) \log \left(\frac{1}{v}\right)+C_{3}\left(\log \frac{1}{v}\right)^{\left(\beta_{0}-1\right) / \beta_{0}}\right) v^{1 / 4}<1 / 2,
$$

where $C_{2}>0$ is the constant in (3.35). Then for $0<t \leq a, r \geq a / C_{*}$ and $\lambda=\frac{\beta_{3}}{8\left(d+\beta_{3}\right)} r,(3.35)$ is less than or equal to

$$
\begin{aligned}
& \frac{s+g}{3}\left(r\left(-1+C_{2}\left(\frac{6}{a \beta_{3}}\left(d+\beta_{3}\right) \log \left(\frac{r}{t}\right)+C_{3}\left(\log \frac{r}{t}\right)^{\left(\beta_{0}-1\right) / \beta_{0}}\right)\left(\frac{t}{r}\right)^{1 / 4}\right)\right) \\
\leq & -\frac{s+g}{6} r \leq-\left(\frac{d}{\beta_{3}}+1\right) \log \frac{r}{t}-\frac{C_{3}}{6}\left(\log \frac{r}{t}\right)^{\left(\beta_{0}-1\right) / \beta_{0}} r .
\end{aligned}
$$

By this inequality, (3.32) and (3.35), we have for $\lambda=\frac{\beta_{3}}{8\left(d+\beta_{3}\right)}|x-y|$ and $t \in(0, a]$,

$$
\begin{aligned}
q^{(\lambda)}(t, x, y) & \leq c_{1} t^{-d / \beta_{3}}\left(\frac{t}{C_{*}|x-y|}\right)^{\frac{d}{\beta_{3}}+1} e^{-c_{8}\left(\log \frac{r}{t}\right)^{\left(\beta_{0}-1\right) / \beta_{0}} r} \\
& \leq c_{9} \frac{t}{|x-y|^{\frac{d}{\beta_{3}}+1}} e^{-c_{8}\left(\log \frac{r}{t}\right)^{\left(\beta_{0}-1\right) / \beta_{0}} r} .
\end{aligned}
$$


It then follows from (3.34) and [3. Lemma 3.2] that for every $t \in(0, a]$ and $|x-y| \geq$ $a / C_{*}$, we have with $\lambda=\frac{\beta_{3}}{8\left(d+\beta_{3}\right)}|x-y| \in(b, \infty)$ that

$$
\begin{aligned}
& q(t, x, y) \\
\leq & q^{(\lambda)}(t, x, y)+t \sup _{\xi \in \mathbb{R}^{d}}\left\|J_{\lambda}(\xi, \cdot)\right\|_{\infty} \\
\leq & \frac{c_{9} t}{|x-y|^{\frac{d}{\beta_{3}}+1}} e^{-c_{8}\left(\log \frac{r}{t}\right)^{\left(\beta_{0}-1\right) / \beta_{0}} r}+\frac{c_{4} t}{\left(\frac{\beta_{3}}{8\left(d+\beta_{3}\right)}|x-y|\right)^{d+\beta_{3}}} e^{-\gamma_{3} c_{5}\left(\frac{\beta_{3}}{8\left(d+\beta_{3}\right)}|x-y|\right)^{\beta_{0}}} \\
\leq & c_{10} t\left(\exp \left(-c_{11}|x-y|(\log (|x-y| / t))^{\left(\beta_{0}-1\right) / \beta_{0}}\right)+\exp \left(-c_{11}|x-y|^{\beta_{0}}\right)\right)
\end{aligned}
$$

This proves (3.28).

The proof of the next theorem is an easy modification of the proof of [10, Theorem 2.3 ], so we skip the proof.

Theorem 3.5. When $\beta_{0}=\infty$, that is, when

$$
\kappa_{1}^{-1} \frac{1}{|x-y|^{d} \phi_{1}(|x-y|)} 1_{\{|x-y| \leq 1\}} \leq J_{0}(x, y) \leq \kappa_{1} \frac{1}{|x-y|^{d} \phi_{2}(|x-y|)} 1_{\{|x-y| \leq 1\}},
$$

we have

$$
p(t, x, y) \leq c_{1} t^{-d / 2} e^{-c_{2}\left(|x-y| \log (|x-y| / t) \wedge|x-y|^{2} / t\right)} \quad \text { when } t>1 .
$$

We now summarize Theorems 3.23 .5 and give the upper bound of Theorems 1.2 and 1.4 .

Theorem 3.6. Suppose the jumping kernel $J_{0}$ satisfies (3.12). In the estimates below for the case of $t \in(0,1]$ and $|x-y| \leq 1$, we assume further that $\phi_{1}=\phi_{2}$. Then there are $c_{k}, k=1, \cdots, 9$, so that the following holds.

(a) When $\beta_{0} \in(0,1]$,

$$
p(t, x, y) \leq c_{1} \begin{cases}\frac{1}{\left(\phi_{1}^{-1}(t)\right)^{d}} \wedge \frac{t}{|x-y|^{d}\left(\phi_{2} \psi_{2}\right)\left(c_{2}|x-y|\right)} & \text { when } t \in(0,1] ; \\ t^{-d / 2} e^{-c_{3}\left(|x-y|^{\beta_{0}} \wedge|x-y|^{2} / t\right)} & \text { when } t>1 .\end{cases}
$$

(b) When $\beta_{0} \in(1, \infty)$,

$$
p(t, x, y) \leq c_{4}\left\{\begin{array}{c}
\frac{1}{\left(\phi_{1}^{-1}(t)\right)^{d}} \wedge \frac{t}{|x-y|^{d} \phi_{1}(|x-y|)} \\
\text { when } t \in(0,1] \text { and }|x-y| \leq 1 ; \\
t\left(e^{-c_{5}|x-y|\left(\log \frac{|x-y|}{t}\right)^{\left(\beta_{0}-1\right) / \beta_{0}}}+e^{-c_{5}|x-y|^{\beta_{0}}}\right) \\
\text { when } t \in(0,1] \text { and }|x-y| \geq 1 ; \\
t^{-d / 2} e^{-c_{6}\left(|x-y|\left(\log \frac{|x-y|}{t}\right)^{\left(\beta_{0}-1\right) / \beta_{0}} \wedge|x-y|^{2} / t\right)} \\
\text { when } t>1 .
\end{array}\right.
$$

(c) When $\beta_{0}=\infty$, that is, when

$$
\kappa_{1}^{-1} \frac{1}{|x-y|^{d} \phi_{1}(|x-y|)} 1_{\{|x-y| \leq 1\}} \leq J_{0}(x, y) \leq \kappa_{1} \frac{1}{|x-y|^{d} \phi_{1}(|x-y|)} 1_{\{|x-y| \leq 1\}},
$$


we have

$$
p(t, x, y) \leq c_{7}\left\{\begin{array}{l}
\frac{1}{\phi_{1}^{-1}(t)^{d}} \wedge \frac{t}{|x-y|^{d} \phi_{1}(|x-y|)} \\
e^{-c_{8}|x-y| \log (|x-y| / t)} \quad \begin{array}{l}
\text { when } t \in(0,1] \text { and }|x-y|<1 ; \\
\text { when } t \in(0,1] \text { and }|x-y| \geq 1 \\
t^{-d / 2} e^{-c_{9}\left(|x-y| \log (|x-y| / t) \wedge|x-y|^{2} / t\right)} \\
\text { when } t>1 .
\end{array}
\end{array}\right.
$$

Proof. The proof of (c) is a combination of Proposition 3.1. Theorem 3.5 and [1], Proposition 2.2]. So we assume $0<\beta_{0}<\infty$. The case $t \geq 1$ is established in Theorems 3.2 3.3. In view of Theorem 3.4 with $a=1$, we only need to consider the case when $t \in(0,1]$ and $|x-y|<1 / C^{*}=: R_{0}$. But by considering the bilinear form $\left(\mathcal{E}^{*}, \mathcal{F}\right)$ with

$$
\begin{aligned}
\mathcal{E}^{*}(v, v)=\int_{\mathbb{R}^{d} \times \mathbb{R}^{d}}(v(\xi)-v(\eta))^{2}\left(J(\xi, \eta) \mathbf{1}_{\{|\xi-\eta|<1\}}\right. \\
\left.+\frac{\kappa_{1}}{|\xi-\eta|^{d} \psi_{2}(|\xi-\eta|)} \mathbf{1}_{\{|\xi-\eta| \geq 1\}}\right) d \eta d \xi,
\end{aligned}
$$

this follows directly from [3, Lemma 3.2] and [11, Proposition 2.2].

\section{Near diagonal lower bound estimate AND PARABOLIC HARNACK INEQUALITY}

4.1. Large time near diagonal lower bound estimate. In this section, we always assume $\beta \in(0, \infty)$ and will give the proof of the near diagonal lower bound estimate of the heat kernel. Note that the case $\beta=\infty$ is proved in [10, Theorem $3.5]$.

Let $\sigma \in(0, \infty)$ and $\mathcal{M}(\sigma)$ be the set of all non-increasing function $\Psi$ from $[0,1]$ to $[0,1]$ such that $\Psi(s)>\Psi(1)=0$ for every $s \in[0,1)$ and

$$
\Psi\left(s+\frac{1}{2}\left((1-s) \wedge \frac{1}{2}\right)\right) \geq \sigma \Psi(s), \quad s \in(0,1) .
$$

We use $\mathcal{N}(\sigma)$ to denote all the functions $\Phi$ of the form $c \Psi(|x|)$ for some $\Psi \in \mathcal{M}(\sigma)$ having $\int_{\mathbb{R}^{d}} \Phi(x) d x=1$. For $\Phi \in \mathcal{N}(\sigma)$, define

$$
u_{\Phi}:=\int_{B(0,1)} u(x) \Phi(x) d x .
$$

The following weighted Poincaré inequality is proved in [10. For every $d \geq 1,0<$ $\alpha<2$ and $\sigma \in(0, \infty)$, there exists a positive constant $c_{1}=c_{1}(d, \alpha, \sigma)$ independent of $r \geq 1$, such that for every $\Phi \in \mathcal{N}(\sigma)$ and $u \in L^{1}(B(0,1), \Phi(x) d x)$,

$$
\begin{aligned}
& \int_{B(0,1)}\left(u(x)-u_{\Phi}\right)^{2} \Phi(x) d x \\
\leq & c_{1} \int_{B(0,1) \times B(0,1)}(u(x)-u(y))^{2} \frac{r^{2-\alpha} \mathbf{1}_{\{|x-y| \leq 1 / r\}}}{|x-y|^{d+\alpha}}(\Phi(x) \wedge \Phi(y)) d x d y .
\end{aligned}
$$

Moreover, it is shown in [10] that the constant $c_{2}$ stays bounded for $\alpha \in(0,2)$. Thus by taking $\alpha \rightarrow 0$, we get the following (more appropriate) form of weighted 
Poincaré inequality. For every $d \geq 1$, there exists a positive constant $c_{2}=c_{2}(d, \sigma)$ independent of $r \geq 1$, such that for every $u \in L^{1}(B(0,1), \Phi(x) d x)$,

$$
\begin{aligned}
& \int_{B(0,1)}\left(u(x)-u_{\Phi}\right)^{2} \Phi(x) d x \\
\leq & c_{2} r^{2} \int_{B(0,1) \times B(0,1)} \frac{(u(x)-u(y))^{2}}{|x-y|^{d}} \mathbf{1}_{\{|x-y| \leq 1 / r\}}(\Phi(x) \wedge \Phi(y)) d x d y .
\end{aligned}
$$

For a non-negative function $\varphi$ on $(0, \infty)$ with $\varphi(0+)=0, \varphi(r)>0$ on $(0,1]$ and $\|\varphi\|_{\infty,[0,1]}:=\sup _{r \in[0,1]}|\varphi(r)|<\infty$, we get from the above inequality that, for every $u \in L^{1}(B(0,1), \Phi(x) d x)$,

$$
\begin{aligned}
& \int_{B(0,1)}\left(u(x)-u_{\Phi}\right)^{2} \Phi(x) d x \\
\leq & c_{2} r^{2} \int_{B(0,1) \times B(0,1)} \frac{(u(x)-u(y))^{2}}{|x-y|^{d}} \mathbf{1}_{\{|x-y| \leq 1 / r\}}(\Phi(x) \wedge \Phi(y)) d x d y \\
\leq & c_{2} r^{2} \cdot\|\varphi\|_{\infty,[0,1]} \int_{B(0,1) \times B(0,1)} \frac{(u(x)-u(y))^{2}}{|x-y|^{d} \varphi(r|x-y|)} \mathbf{1}_{\{|x-y| \leq 1 / r\}}(\Phi(x) \wedge \Phi(y)) d x d y \\
\leq & c_{2} r^{2} \cdot\|\varphi\|_{\infty,[0,1]} \int_{B(0,1) \times B(0,1)} \frac{(u(x)-u(y))^{2}}{|x-y|^{d} \varphi(r|x-y|)}(\Phi(x) \wedge \Phi(y)) d x d y .
\end{aligned}
$$

Thus we have

Theorem 4.1. For every $d \geq 1$ and $\sigma \in(0, \infty)$, there is a positive constant $c=$ $c(d, \sigma)$ independent of $r>1$, such that for every $\Phi \in \mathcal{N}(\sigma), u \in L^{1}(B(0,1), \Phi d x)$ and a non-negative function $\varphi$ on $(0, \infty)$ with $\varphi(0+)=0, \varphi(r)>0$ on $(0,1]$ and $\|\varphi\|_{\infty,[0,1]}<\infty$,

$$
\begin{aligned}
& \int_{B(0,1)}\left(u(x)-u_{\Phi}\right)^{2} \Phi(x) d x \\
\leq & c r^{2} \cdot\|\varphi\|_{\infty,[0,1]} \int_{B(0,1) \times B(0,1)} \frac{(u(x)-u(y))^{2}}{|x-y|^{d} \varphi(r|x-y|)}(\Phi(x) \wedge \Phi(y)) d x d y .
\end{aligned}
$$

Here $u_{\Phi}:=\int_{B(0,1)} u(x) \Phi(x) d x$.

Now let's consider $(\mathcal{Q}, \mathcal{D})$ of (3.1) and assume that the jump kernel $J_{0}(x, y)$ for $(\mathcal{E}, \mathcal{F})$ satisfies (3.12). Recall that $q(t, x, y)$ is the transition density function for the associated Hunt process $Y$ with respect to the Lebesgue measure on $\mathbb{R}^{d}$.

Let $J_{0}(x, y)$ be the function satisfying (3.9) $-(3.12)$. For $\delta \in(0,1)$, set

$$
\widehat{J}_{\delta}(x, y)=J_{0}(x, y) \mathbf{1}_{\{|x-y| \geq \delta\}}+\kappa_{1} \frac{1}{|x-y|^{d} \phi_{2}(|x-y|) \psi_{2}(|x-y|)} \mathbf{1}_{\{|x-y|<\delta\}},
$$

and define $\left(\mathcal{E}^{\delta}, \mathcal{F}^{\delta}\right)$ in the same way as we defined $(\mathcal{Q}, \mathcal{D})$ in (3.1) but with $\widehat{J}_{\delta}$ in place of $J_{0}$.

For $\delta \in(0,1)$, let $\widehat{Y}^{\delta}$ be the symmetric Markov process associated with $\left(\mathcal{E}^{\delta}, \mathcal{F}^{\delta}\right)$. By [20, Theorem 3.1], the process $\widehat{Y}^{\delta}$ is conservative. Moreover, by Proposition 3.1, there is a properly $\mathcal{E}^{\delta}$-exceptional set $\mathcal{N}_{\delta} \subset \mathbb{R}^{d}$ and a positive symmetric kernel $q^{\delta}(t, x, y)$ defined on $[0, \infty) \times\left(\mathbb{R}^{d} \backslash \mathcal{N}_{\delta}\right) \times\left(\mathbb{R}^{d} \backslash \mathcal{N}_{\delta}\right)$ such that for every $x \in \mathbb{R}^{d} \backslash \mathcal{N}_{\delta}$ and $s, t>0$,

$$
\mathbb{E}_{x}\left[f\left(\widehat{Y}_{t}^{\delta}\right)\right]=\int_{\mathbb{R}^{d}} q^{\delta}(t, x, y) f(y) d y, \quad q^{\delta}(t+s, x, y)=\int_{\mathbb{R}^{d}} q^{\delta}(t, x, z) q^{\delta}(s, z, y) d z,
$$


and for every $x, y \in \mathbb{R}^{d} \backslash \mathcal{N}_{\delta}$,

$$
q^{\delta}(t, x, y) \leq c_{\delta} t^{-d / 2}
$$

Moreover, there is an $\mathcal{E}^{\delta}$-nest $\left\{F_{k}^{\delta}, k \geq 1\right\}$ of compact subsets of $\mathbb{R}^{d}$ so that $\mathcal{N}_{\delta}=$ $\mathbb{R}^{d} \backslash \bigcup_{k=1}^{\infty} F_{k}^{\delta}$ and that for every $t>0$ and $y \in \mathbb{R}^{d} \backslash \mathcal{N}_{\delta}, x \mapsto q^{\delta}(t, x, y)$ is continuous on each $F_{k}^{\delta}$. For ball $B\left(x_{0}, r\right) \subset \mathbb{R}^{d}$, let $q^{\delta, B\left(x_{0}, r\right)}(t, x, y)$ denote the transition density function of the subprocess $\widehat{Y}^{\delta, B\left(x_{0}, r\right)}$ of $\widehat{Y}^{\delta}$ killed upon leaving the ball $B\left(x_{0}, r\right)$.

We consider the bilinear form $\left(\mathcal{E}^{(1), \delta}, \mathcal{F}^{\delta}\right)$ with

$$
\mathcal{E}^{(1), \delta}(v, v):=\int_{\mathbb{R}^{d}} \int_{\mathbb{R}^{d}}(v(\xi)-v(\eta))^{2} \widehat{J}_{\delta}^{(1)}(\xi, \eta) d \eta d \xi,
$$

where

$$
\widehat{J}_{\delta}^{(1)}(\xi, \eta):=\mathbf{1}_{\{|\xi-\eta| \leq 1\}} \widehat{J}_{\delta}(\xi, \eta) .
$$

Let $\widehat{Y}^{(1), \delta}$ be the corresponding Hunt process associated with the regular Dirichlet form $\left(\mathcal{E}^{(1), \delta}, \mathcal{F}^{\delta}\right)$. Since $\widehat{Y}^{(1), \delta}$ can be obtained from $\widehat{Y}^{\delta}$ by removing its jumps of size larger than $1, \widehat{Y}^{(1), \delta}$ can start from every point in $\mathbb{R}^{d} \backslash \mathcal{N}_{\delta}$. Moreover, $\widehat{Y}^{(1), \delta}$ admits a quasi-continuous transition density function $q^{(1), \delta}(t, \xi, \eta)$ on $(0, \infty) \times\left(\mathbb{R}^{d} \backslash\right.$ $\left.\mathcal{N}_{\delta}\right) \times\left(\mathbb{R}^{d} \backslash \mathcal{N}_{\delta}\right)$ with respect to the Lebesgue measure that has similar properties as that for $q^{\delta}(t, x, y)$. Let $q^{(1), \delta, D}(t, \xi, \eta)$ be the transition density function of the subprocess $\widehat{Y}^{(1), \delta, D}$ of $\widehat{Y}^{(1), \delta}$ killed upon leaving an open set $D$. It follows from [1, Lemma 3.6] that for $x, y \in \mathbb{R}^{d} \backslash \mathcal{N}_{\delta}$,

$$
q^{\delta}(t, x, y) \geq e^{-t\left\|\mathcal{J}_{\delta}\right\|_{\infty}} q^{(1), \delta}(t, x, y) \quad \text { and } \quad q^{\delta, D}(t, x, y) \geq e^{-s\left\|\mathcal{J}_{\delta}\right\|_{\infty}} q^{(1), \delta, D}(t, x, y),
$$

where $\mathcal{J}_{\delta}(x):=\int_{\mathbb{R}^{d}} \widehat{J}_{\delta}(x, y) \mathbf{1}_{\{|x-y|>1\}} d y$. Since $\sup _{\delta \in(0,1)}\left\|\mathcal{J}_{\delta}\right\|_{\infty}<\infty$, using (4.4) the next three results follow from [1, Proposition 4.3, Lemma 4.5, Lemma 4.6], respectively.

Proposition 4.2. There is a constant $c_{1}=c_{1}(\delta, r)>0$ such that for every $t \in$ $\left[r^{2} / 8, r^{2} / 4\right]$ and $x, y \in B\left(x_{0}, r\right) \backslash \mathcal{N}_{\delta}$,

$$
q^{\delta, B\left(x_{0}, r\right)}(t, x, y) \geq c_{1}(r-|x|)^{\beta_{4}}(r-|y|)^{\beta_{4}} .
$$

Let $\left(\mathcal{E}^{\delta}, \mathcal{F}^{\delta, B\left(x_{0}, r\right)}\right)$ be the Dirichlet form for the killed process $\widehat{Y}^{\delta, B\left(x_{0}, r\right)}$.

Lemma 4.3. For every $t>0$ and $y_{0} \in B\left(x_{0}, r\right) \backslash \mathcal{N}_{\delta}$, it holds that $q^{\delta, B\left(x_{0}, r\right)}\left(t, x, y_{0}\right)$ $\in \mathcal{F}^{\delta, B\left(x_{0}, r\right)}$.

Define

$$
\Phi_{r}(x)=\left(r^{2}-|x|^{2}\right)^{12 /\left(2-\beta_{4}\right)} \mathbf{1}_{B\left(x_{0}, r\right)}(x) .
$$

Lemma 4.4. For every $t>0$ and $y_{0} \in B\left(x_{0}, r\right) \backslash \mathcal{N}_{\delta}$, it holds that

$$
\Phi_{r}(\cdot) / q^{\delta, B\left(x_{0}, r\right)}\left(t, x, y_{0}\right) \in \mathcal{F}^{\delta, B\left(x_{0}, r\right)} .
$$

Let $q_{r}^{B\left(x_{0}, 1\right)}(t, x, y):=r^{d} q^{\delta, B\left(x_{0}, r\right)}\left(r^{2} t, r x, r y\right)$ and $x_{1} \in B\left(x_{0}, 1\right)$, and define

$$
\begin{aligned}
u_{r}(t, x) & :=q_{r}^{B\left(x_{0}, 1\right)}\left(t, x, x_{1}\right), \\
G(t) & :=\int_{B\left(x_{0}, 1\right)} \Phi_{1}(y) \log u_{r}(t, y) d y-\frac{1}{2} \int_{B\left(x_{0}, 1\right)} \Phi_{1}(x) \log \Phi_{1}(x) d x .
\end{aligned}
$$


Using the above three results, the proof of the next result is almost identical to that of [1, Lemma 4.7]. So we skip the proof.

\section{Lemma 4.5.}

$$
G^{\prime}(t)=-\mathcal{E}^{(r)}\left(u_{r}(t, \cdot), \frac{\Phi_{1}}{u_{r}(t, \cdot)}\right)
$$

where

$$
\mathcal{E}^{(r)}(u, u):=\int_{\mathbb{R}^{d} \times \mathbb{R}^{d}}(u(x)-u(y))^{2} r^{d+2} \widehat{J}_{\delta}(r x, r y) d x d y .
$$

The idea of the proof of the following theorem is motivated by that of [1, Proposition 4.9] and [10, Theorem 3.4].

Theorem 4.6. For each $\delta_{0}>0$ there exists $c=c\left(\delta_{0}, \beta, \beta_{0}\right)>0$, independent of $\delta \in(0,1)$, such that for every $x_{0} \in \mathbb{R}^{d}$,

(4.6) $q^{\delta}(t, x, y) \geq c t^{-d / 2} \quad$ for every $t \geq \delta_{0}$ and $x, y \in \mathbb{R}^{d} \backslash \mathcal{N}_{\delta}$ with $|x-y|^{2} \leq t$.

Moreover, for each $\delta_{0}>0$ and $0<\varepsilon<M<\infty$ there exists $c=c\left(\delta_{0}, \varepsilon, M, \beta, \beta_{0}\right)>$ 0 , independent of $\delta \in(0,1)$, such that for every $x_{0} \in \mathbb{R}^{d}, t \geq \delta_{0}, s \in[\varepsilon t, M t]$ and every $x, y \in B\left(x_{0}, 3 \sqrt{t} / 4\right) \backslash \mathcal{N}_{\delta}$,

$$
q^{\delta, B\left(x_{0}, t^{1 / 2}\right)}(s, x, y) \geq c t^{-d / 2} .
$$

Proof. Fix $\delta \in(0,1)$ and, for simplicity, in this proof we sometimes drop the superscript " $\delta$ " from $\widehat{Y}^{\delta}$ and $q^{\delta}(t, x, y)$. Recall that for the ball $B_{r}:=B(0, r) \subset \mathbb{R}^{d}$, $q^{B_{r}}(t, x, y)$ is the transition density function of the subprocess $\widehat{Y}^{B_{r}}$ of $\widehat{Y}$ killed upon leaving the ball $B_{r}$. It follows from Lemmas 4.3 and 4.4 that for every $t>0$ and $y_{0} \in B_{r}, q^{B_{r}}\left(t, x, y_{0}\right) \in \mathcal{F}^{B_{r}}$ and $\varphi_{r}(\cdot) / q^{B_{r}}\left(t, x, y_{0}\right) \in \mathcal{F}^{B_{r}}$, where $\left(\mathcal{E}, \mathcal{F}^{B_{r}}\right)$ is the Dirichlet form for the killed process $\widehat{Y}^{B_{r}}$.

Note that the Dirichlet form of $\left\{r^{-1} \widehat{Y}_{r^{2} t}, t \geq 0\right\}$ is $\left(\mathcal{E}^{(r)}, \mathcal{F}^{(r)}\right)$, where

$$
\begin{aligned}
\mathcal{E}^{(r)}(u, u) & =\int_{\mathbb{R}^{d} \times \mathbb{R}^{d}}(u(x)-u(y))^{2} r^{d+2} \widehat{J}_{\delta}(r x, r y) d x d y \\
\mathcal{F}^{(r)} & =\left\{u \in L^{2}(u, u): \mathcal{E}^{(r)}(u, u)<\infty\right\}=W^{\beta_{4} / 2,2}\left(\mathbb{R}^{d}\right) .
\end{aligned}
$$

For $u \in L^{2}\left(\mathbb{R}^{d}, d x\right)$, its Fourier transform $\widehat{u}$ is defined by

$$
\widehat{u}(\xi):=(2 \pi)^{-d / 2} \int_{\mathbb{R}^{d}} e^{i \xi \cdot y} u(y) d y .
$$

Then

$$
\begin{aligned}
\mathcal{E}_{1}^{(r)}(u, u) & \leq c_{0}\left(\int_{\mathbb{R}^{d} \times \mathbb{R}^{d}} \frac{(u(x)-u(y))^{2}}{|x-y|^{d+\beta_{4}}} d x d y+\int_{\mathbb{R}^{d}} u(x)^{2} d x\right) \\
& =c_{1} \int_{\mathbb{R}^{d}}\left(|\xi|^{\beta_{4}}+1\right)|\widehat{u}(\xi)|^{2} d \xi \\
& \leq c_{2} \int_{\mathbb{R}^{d}}\left(|\xi|^{2}+1\right)|\widehat{u}(\xi)|^{2} d \xi=c_{3} \int_{\mathbb{R}^{d}}\left(|\nabla u(x)|^{2}+u(x)^{2}\right) d x
\end{aligned}
$$

Define

$$
q_{r}^{B}(t, x, y):=r^{d} q^{B_{r}}\left(r^{2} t, r x, r y\right) \quad \text { for } t>0 \text { and } x, y \in B \backslash \mathcal{N}_{\delta} .
$$

Note that $q_{r}^{B}(t, x, y)$ is the transition density function for the process $r^{-1} \widehat{Y}_{r^{2} t}^{B_{r}}$. The latter is the subprocess of $\left\{r^{-1} \widehat{Y}_{r^{2} t}, t \geq 0\right\}$ killed upon leaving the unit ball $B(0,1)$, 
whose Dirichlet form will be denoted as $\left(\mathcal{E}^{(r)}, \mathcal{F}^{(r), B}\right)$. It follows from Proposition 4.2 that there is a constant $c_{4}=c_{4}(\delta, r)>0$ such that

$q_{r}^{B}(t, x, y) \geq c_{4}(1-|x|)^{\beta_{4}}(1-|y|)^{\beta_{4}} \quad$ for every $t \in[1 / 8,1 / 4]$ and $x, y \in B(0,1) \backslash \mathcal{N}_{\delta}$.

Let $\Phi(x):=c_{5} \Phi_{1}(x)$, where $\Phi_{1}$ is the function defined in (4.5) and $c_{5}$ is a normalizing constant so that $\int_{\mathbb{R}^{d}} \Phi(x) d x=1$. Let $x_{0} \in B(0,1) \backslash \mathcal{N}_{\delta}$ and define

$$
\begin{aligned}
u(t, x) & :=q_{r}^{B}\left(t, x, x_{0}\right), \quad v(t, x):=q_{r}^{B}\left(t, x, x_{0}\right) / \Phi(x)^{1 / 2}, \\
H(t) & :=\int_{B(0,1)} \Phi(y) \log u(t, y) d y, \\
G(t) & :=\int_{B(0,1)} \Phi(y) \log v(t, y) d y=H(t)-c_{6} .
\end{aligned}
$$

The remainder of the argument does not use the condition on $\hat{J}_{\delta}$, and, in particular, the constants can be taken to be independent of $\delta \in(0,1)$.

Let $q_{r}(t, x, y):=r^{d} q\left(r^{2} t, r x, r y\right)$, which is the transition density function with respect to the Lebesgue measure on $\mathbb{R}^{d}$ for the symmetric jump process $\widehat{Y}_{t}^{(r)}:=$ $r^{-1} \widehat{Y}_{r^{2} t}$, whose jumping intensity function is $r^{d+2} J(r x, r y)$. Let $x \in \mathbb{R}^{d} \backslash \mathcal{N}_{\delta}$. Using Theorem 3.2 for $r^{2} t \geq 1$ and $\beta_{0}>1$,

$$
\begin{aligned}
& \mathbb{P}_{x}\left(\widehat{Y}_{t}^{(r)} \notin B(x, 1 / 4)\right) \\
= & \int_{B(x, 1 / 4)^{c}} r^{d} q\left(r^{2} t, r x, r y\right) d y=\int_{B(r x, r / 4)^{c}} q\left(r^{2} t, r x, z\right) d z \\
\leq & c_{7} \int_{\left\{w \in \mathbb{R}^{d}:|w| \geq\left((r / 4) \vee\left(r^{2} t\right)\right)\right\}} e^{-c_{8}|w|\left(\log |w| /\left(r^{2} t\right)\right)^{\left(\beta_{0}-1\right) / \beta_{0}}} d w \\
& +c_{9} \int_{r / 4}^{r^{2} t} r^{-d} t^{-d / 2} \exp \left(-\frac{c_{10} s^{2}}{r^{2} t}\right) s^{d-1} d s \\
\leq & c_{7} \int_{\left\{w \in \mathbb{R}^{d}:|w| \geq r / 4\right\}} e^{-c_{11}|w|} d w+c_{9} \int_{1 /(4 \sqrt{t})}^{\infty} \exp \left(-c_{10} u^{2}\right) u^{d-1} d u .
\end{aligned}
$$

Similarly, using Theorem 3.3, for $r^{2} t \geq 1$ and $\beta_{0} \leq 1$,

$$
\begin{aligned}
& \mathbb{P}_{x}\left(\widehat{Y}_{t}^{(r)} \notin B(x, 1 / 4)\right) \\
\leq & c_{12} \int_{\left\{w \in \mathbb{R}^{d}:|w| \geq r / 4\right\}} e^{-c_{13}|w|_{0}^{\beta}} d w+c_{14} \int_{1 /(4 \sqrt{t})}^{\infty} \exp \left(-c_{15} u^{2}\right) u^{d-1} d u .
\end{aligned}
$$

For each $\beta_{0}>0$, using (4.11)-(4.12) we choose that $t_{0} \in(0,1 / 2)$ be small so that $\mathbb{P}_{x}\left(\widehat{Y}_{t}^{(r)} \notin B(x, 1 / 4)\right)<1 / 16+1 / 16=1 / 8 \quad$ for every $r \geq t_{0}^{-1 / 2}$ and $0<t \leq t_{0}$.

By Lemma 3.8 of [1], we have that for every $r \geq t_{0}^{-1 / 2}$,

$$
\mathbb{P}_{x}\left(\sup _{s \in\left[0, t_{0}\right]}\left|\widehat{Y}_{s}^{(r)}-\widehat{Y}_{0}^{(r)}\right|>1 / 4\right) \leq 1 / 4 .
$$


Write $\widehat{J}^{(r)}(x, y):=r^{d+2} \widehat{J}_{\delta}(r x, r y)$ and $\kappa_{B}^{(r)}(x):=2 \int_{\mathbb{R}^{d} \backslash B(0,1)} \widehat{J}^{(r)}(x, y) d y$ for $x \in B:=B(0,1)$. Then we have from (4.8) and Lemma 4.5.

$$
\begin{aligned}
G^{\prime}(t)=-\int_{B} & \int_{B} \frac{[u(t, y)-u(t, x)]}{u(t, x) u(t, y)}[u(t, x) \Phi(y)-\Phi(x) u(t, y)] \widehat{J}^{(r)}(x, y) d y d x \\
& -\int_{B} \Phi(x) \kappa_{B}^{(r)}(x) d x .
\end{aligned}
$$

The main step is to show that for all $t$ in $(0,1]$ and $r \geq t_{0}^{-1 / 2}$ one has

$$
G^{\prime}(t) \geq-c_{16}+c_{17} \int_{B}(\log u(t, y)-H(t))^{2} \Phi(y) d y
$$

for positive constants $c_{16}, c_{17}$. By [10, (3.13)] and the argument that follows it, we have

$$
\begin{aligned}
& \frac{[u(t, y)-u(t, x)]}{u(t, x) u(t, y)}[u(t, x) \Phi(y)-\Phi(x) u(t, y)] \\
\leq & \left(\Phi(x)^{1 / 2}-\Phi(y)^{1 / 2}\right)^{2}-\sqrt{\Phi(x) \Phi(y)}(\log v(t, y)-\log v(t, x))^{2} .
\end{aligned}
$$

Substituting in the formula for $G^{\prime}(t)$,

$$
\begin{aligned}
& H^{\prime}(t)=G^{\prime}(t) \\
& \geq-c_{18}+\int_{B} \int_{B}(\log v(t, y)-\log v(t, x))^{2} \sqrt{\Phi(x) \Phi(y)} J^{(r)}(x, y) d x d y \\
& \geq-c_{18}+r^{2} \int_{B} \int_{B}(\log v(t, y)-\log v(t, x))^{2}(\Phi(x) \wedge \Phi(y)) \frac{1}{|x-y|^{d} \varphi(r|x-y|)} d x d y,
\end{aligned}
$$

where

$$
\varphi(s):= \begin{cases}\left(\kappa_{0}\right)^{-1} s^{\beta_{2}} & \text { when } s<\delta \\ \kappa_{1} \phi(s) & \text { when } s>\delta .\end{cases}
$$

Thus, using Theorem 4.1, for all $t$ in $(0,1]$ and $r \geq t_{0}^{-1 / 2}$,

$$
\begin{aligned}
H^{\prime}(t) & \geq-c_{18}+c_{19} \int_{B}(\log v(t, y)-G(t))^{2} \Phi(y) d y \\
& \geq-c_{20}+c_{21} \int_{B}(\log u(t, y)-H(t))^{2} \Phi(y) d y
\end{aligned}
$$

which gives (4.14). Note that in the first inequality we used the fact that

$$
\begin{aligned}
& \int_{B} \int_{B}\left(\Phi(x)^{1 / 2}-\Phi(y)^{1 / 2}\right)^{2} J^{(r)}(x, y) d x d y+\int_{B} \Phi(x) \kappa_{B}^{(r)}(x) d x \\
= & \mathcal{E}^{(r)}\left(\Phi^{1 / 2}, \Phi^{1 / 2}\right)<\infty,
\end{aligned}
$$

which follows from (4.9).

Now following the argument of the proof of [10, Theorem 3.4, pages 851-853] we conclude that

$$
q(t, x, y) \geq q^{B\left(x_{0}, \sqrt{t}\right)}(t, x, y) \geq c_{22} t^{-d / 2}
$$

for every $t \geq t_{0}^{-1}$ and $x, y \in \mathbb{R}^{d} \backslash \mathcal{N}_{\delta}$ with $|x-y|^{2} \leq t$, and

$$
q^{B\left(x_{0}, \sqrt{t}\right)}(s, x, y) \geq c_{23} t^{-d / 2}
$$

for $t \geq t_{0}^{-1}, x, y \in B\left(x_{0}, 3 \sqrt{t} / 4\right) \backslash \mathcal{N}_{\delta}$ and $s \in[\varepsilon t, M t]$. 
Next suppose that $\delta_{0}<t_{0}^{-1}$ and $\delta_{0} \leq t \leq t_{0}^{-1}$. Let $n_{0}=1+\left[2 / \sqrt{t_{0} \delta_{0}}\right]$, where $[a]$ is the largest integer which is no larger than $a$. By [1, Theorem 4.10] and our (4.4) we have

$q^{B\left(x_{0}, \sqrt{\delta_{0}}\right)}(u, x, y) \geq c_{24}, \quad$ for every $\frac{\varepsilon \delta_{0}}{2 n_{0}} \leq u \leq 2 t_{0}^{-1}$ and $x, y \in B\left(x_{0}, 3 \sqrt{\delta_{0}} / 4\right) \backslash \mathcal{N}_{\delta}$, where the constant $c_{24}$ is independent of $\delta$ and $x_{0} \in \mathbb{R}^{d}$. Given

$$
x, y \in B\left(x_{0}, 3 \sqrt{t} / 4\right) \backslash \mathcal{N}_{\delta},
$$

let $z_{1} \cdots z_{n_{0}-1}$ be equally spaced points on the line segment joining $x$ and $y$ such that $x \in B\left(z_{1}, 3 \sqrt{\delta_{0}} / 4\right) \subset B\left(z_{1}, \sqrt{\delta_{0}}\right) \subset B\left(x_{0}, \sqrt{t}\right)$ and $y \in B\left(z_{n_{0}-1}, 3 \sqrt{\delta_{0}} / 4\right) \subset$ $B\left(z_{n_{0}-1}, \sqrt{\delta_{0}}\right) \subset B\left(x_{0}, \sqrt{t}\right)$. Using (4.15) and the semigroup property, we have

$$
\begin{aligned}
& \int_{B\left(x_{0}, \sqrt{t}\right)} \ldots \int_{B\left(x_{0}, \sqrt{t}\right)} q^{B\left(x_{0}, \sqrt{t}\right)}\left(s / n_{0}, x, w_{1}\right) \\
& \ldots q^{B\left(x_{0}, \sqrt{t}\right)}\left(s / n_{0}, w_{n_{0}-1}, y\right) d w_{1} \ldots d w_{n_{0}-1} \\
\geq & \int_{B\left(z_{1}, 3 \sqrt{\delta_{0}} / 4\right)} \ldots \int_{B\left(z_{n_{0}-1}, 3 \sqrt{\delta_{0}} / 4\right)} q^{B\left(z_{1}, \sqrt{\delta_{0}}\right)}\left(s / n_{0}, x, w_{1}\right) \\
\ldots & \ldots q^{B\left(z_{n_{0}}, \sqrt{\delta_{0}}\right)}\left(s / n_{0}, w_{n_{0}-1}, y\right) d w_{1} \ldots d w_{n_{0}-1} \\
\geq & c_{25} \geq c_{25} \delta_{0}^{d / 2} t^{-d / 2} .
\end{aligned}
$$

A similar argument gives (4.6) when $\delta_{0}<t_{0}^{-1}$ and $t \in\left[\delta_{0}, t_{0}^{-1}\right]$.

For any ball $B \subset \mathbb{R}^{d}$, let $\left(\mathcal{E}^{\delta, B}, \mathcal{F}^{\delta, B}\right)$ denote the Dirichlet form of the subprocess $\widehat{Y}^{\delta, B}$ of $\widehat{Y}^{\delta}$ killed upon leaving the ball $B$. It is easy to see that $C_{c}^{1}\left(\mathbb{R}^{d}\right)$ is a common core for $\left(\mathcal{E}^{\delta}, \mathcal{F}^{\delta}\right)$ and $(\mathcal{E}, \mathcal{F})$ and that $C_{c}^{1}(B) \subset C_{c}^{1}\left(\mathbb{R}^{d}\right)$ is a common core for $\left(\mathcal{E}^{\delta, B}, \mathcal{F}^{\delta, B}\right)$ and $\left(\mathcal{E}^{B}, \mathcal{F}^{B}\right)$. Thus by [1, Theorems 2.3 and 2.4] we have the following. (See [1, Definition 2.1] for the definition of Mosco convergence.)

Theorem 4.7. Let $B$ be a ball in $\mathbb{R}^{d}$. Then $\left(\mathcal{E}^{\delta}, \mathcal{F}^{\delta}\right)$ and $\left(\mathcal{E}^{\delta, B}, \mathcal{F}^{\delta, B}\right)$ converge as $\delta \rightarrow 0$ to $(\mathcal{E}, \mathcal{F})$ and $\left(\mathcal{E}^{B}, \mathcal{F}^{B}\right)$, respectively, in the sense of Mosco.

The above theorem implies that the semigroup of $\widehat{Y}^{\delta}$ and $\widehat{Y}^{\delta, B}$ converge in $L^{2}$ to that of $\widehat{Y}$ and $\widehat{Y}^{B}$, respectively. By the same proof as that for [1, Theorem 1.3], we deduce from Theorem 4.6 the following lower bound estimate for the heat kernel of $\widehat{Y}$ and $\widehat{Y}^{B}$.

Recall that $\mathcal{N}$ is the properly exceptional set in Proposition 3.1 .

Theorem 4.8. Suppose the Dirichlet form $(\mathcal{Q}, \mathcal{D})$ is given by (3.1) with the jumping kernel satisfying (3.12). For each $t_{0}>0$ there exists $c_{1}=c_{1}\left(t_{0}\right)>0$ such that for every $x_{0} \in \mathbb{R}^{d}, t \geq t_{0}$,

$$
q(t, x, y) \geq c_{1} t^{-d / 2} \quad \text { for every } x, y \in \mathbb{R}^{d} \backslash \mathcal{N} \text { with }|x-y|^{2} \leq t .
$$

Moreover, for each $t_{0}>0$ and $0<\varepsilon<M<\infty$ there exists $c_{2}=c_{2}\left(t_{0}, \varepsilon, M\right)>0$ such that for every $x_{0} \in \mathbb{R}^{d}, t \geq t_{0}$,

$$
q^{B\left(x_{0}, \sqrt{t}\right)}(s, x, y) \geq c_{2} t^{-d / 2}
$$

for every $s \in[\varepsilon t, M t]$ and q.e. $x, y \in B\left(x_{0}, 3 \sqrt{t} / 4\right) \backslash \mathcal{N}$. 
4.2. Parabolic Harnack inequality. In this section we always assume $\beta_{0} \in$ $(0, \infty]$.

We first introduce a space-time process $Z_{s}:=\left(V_{s}, Y_{s}\right)$, where $V_{s}=V_{0}-s$. The augmented filtration generated by $Z$ will be denoted as $\left\{\widetilde{\mathcal{F}}_{s} ; s \geq 0\right\}$. The law of the space-time process $s \mapsto Z_{s}$ starting from $(t, x)$ will be denoted as $\mathbb{P}_{(t, x)}$.

For each $A \subset[0, \infty) \times \mathbb{R}^{d}$, denote $\sigma_{A}:=\inf \left\{t>0: Z_{t} \in A\right\}$ and $\tau_{A}=\tau(A):=$ $\inf \left\{t>0: Z_{t} \notin A\right\}$ and define $U(t, x, r)=\{t\} \times B(x, r)$. Recall that $\mu_{d+1}$ is the Lebesgue measure on $\mathbb{R}^{d+1}$.

Lemma 4.9. Suppose the Dirichlet form $(\mathcal{Q}, \mathcal{D})$ is given by (3.1) with the jumping kernel $J_{0}$ satisfying condition (3.12). For every $\delta \in(0,1)$ and $R_{*}>0$ there exist $C_{2}, C_{3}>0$ depending $\delta, R_{*} \beta$ and $\beta_{0}$ such that the following are true.

(i) For all $x \in \mathbb{R}^{d} \backslash \mathcal{N}, r \geq R_{*}, t \geq \delta r^{2}$ and any compact subset $A \subset\left[t-\delta r^{2}, t-\right.$ $\left.\delta r^{2} / 2\right] \times B\left(x, \frac{3 r}{4}\right)$,

$$
\mathbb{P}_{(t, x)}\left(\sigma_{A}<\tau_{\left[t-\delta r^{2}, t\right] \times B(x, r)}\right) \geq C_{2} \frac{\mu_{d+1}(A)}{r^{d+2}} .
$$

(ii) For every $R \geq R_{*}, r \in(0, R / 2], x_{0} \in \mathbb{R}^{d} \backslash \mathcal{N}, t \leq 2 R^{2}$ and $\left(t^{\prime}, x\right),(t, z) \in$ $\left[t-3 \delta R^{2}, t\right] \times B\left(x_{0}, R\right)$ with $t^{\prime} \leq t-\delta R^{2} / 2$,

$$
\mathbb{P}_{(t, z)}\left(\sigma_{U\left(t^{\prime}, x, r\right)}<\tau_{\left[t-3 \delta R^{2}, t\right] \times B\left(x_{0}, 2 R\right)}\right) \geq C_{3} \frac{r^{d+2}}{R^{d+2}} .
$$

Proof. (i) Let $\tau_{r}:=\tau_{\left[t-\delta r^{2}, t\right] \times B(x, r)}$ and $A_{s}:=\left\{y \in \mathbb{R}^{d}:(s, y) \in A\right\}$. Note that

$$
\begin{aligned}
& \mathbb{E}_{(t, x)}\left[\int_{0}^{\tau_{r}} \mathbf{1}_{A}\left(t-s, Y_{s}\right) d s\right]=\int_{0}^{\delta r^{2}} \mathbb{P}_{(t, x)}\left(\int_{0}^{\tau_{r}} \mathbf{1}_{A}\left(t-s, Y_{s}\right) d s>u\right) d u \\
\leq & \int_{0}^{\delta r^{2}} \mathbb{P}_{(t, x)}\left(\int_{0}^{\tau_{r}} \mathbf{1}_{A}\left(t-s, Y_{s}\right) d s>0\right) d u \leq \delta r^{2} \mathbb{P}_{(t, x)}\left(\sigma_{A}<\tau_{r}\right) .
\end{aligned}
$$

On the other hand,

$$
\begin{aligned}
& \mathbb{E}_{(t, x)}\left[\int_{0}^{\tau_{r}} \mathbf{1}_{A}\left(t-s, Y_{s}\right) d s\right]=\int_{2^{-1} \delta r^{2}}^{\delta r^{2}} \mathbb{P}_{(t, x)}\left(\left(t-s, Y_{s}^{B(x, r)}\right) \in A\right) d s \\
= & \int_{2^{-1} \delta r^{2}}^{\delta r^{2}} \mathbb{P}_{x}\left(Y_{s}^{B(x, r)} \in A_{t-s}\right) d s=\int_{2^{-1} \delta r^{2}}^{\delta r^{2}} \int_{A_{t-s}} p^{B(x, r)}(s, x, y) d y d s .
\end{aligned}
$$

From [10, Theorem 3.5] and our (4.16), we have for $(s, y) \in\left(2^{-1} \delta r^{2}, \delta r^{2}\right) \times$ $(B(x, 3 r / 4) \backslash \mathcal{N}), p^{B(x, r)}(s, x, y) \geq c_{1} r^{-d}$. Thus

$$
\mathbb{E}_{(t, x)}\left[\int_{0}^{\tau_{r}} \mathbf{1}_{A}\left(t-s, Y_{s}\right) d s\right] \geq c_{1} \int_{2^{-1} \delta r^{2}}^{\delta r^{2}} \int_{A_{t-s}} r^{-d} d y d s \geq c_{1} \mu_{d+1}(A) \frac{1}{r^{d}} .
$$

Combining the above two inequalities we obtain (i).

(ii) Given (4.16), the proof is almost the same as that of [11, Lemma 2.6 (ii)], so we omit it.

Now we consider the symmetric jump process $Y$ whose jumping measure $J$ is given by (1.6). In this case, it follows from [11, Theorem 3.1] that $Y$ admits the jointly continuous transition density function $p(t, x, y)$ and that $Y$ can be modified to start from every point in $\mathbb{R}^{d}$ in view of Meyer's construction (so the properly 
exceptional set $\mathcal{N}$ of $Y$ can be taken to be the empty set). Throughout the remainder of this paper, we consider the space-time process $Z_{s}=\left(V_{0}-s, Y_{s}\right)$, where the jumping kernel $J$ of $Y$ is given by (1.6) and satisfies UJS. We point out that UJS is only used in the next lemma.

Note that for $Q:=[a, b] \times B, \tau_{Q}=\inf \left\{s \geq 0: Z_{s} \notin Q\right\}=\tau_{B} \wedge\left(V_{0}-a\right)_{+} \mathbf{1}_{\left\{V_{0} \leq b\right\}}$.

Lemma 4.10. Let $R_{*}>0, \delta>0$ and $0<a<1 / 3$. For $R \geq R_{*}$, let $Q_{1}=$ $\left[t_{0}, t_{0}+4 \delta R^{2}\right] \times B\left(x_{0}, 3 a R / 2\right), Q_{2}=\left[t_{0}, t_{0}+4 \delta R^{2}\right] \times B\left(x_{0}, 2 a R\right)$ and define

$Q_{-}=\left[t_{0}+\delta R^{2}, t_{0}+2 \delta R^{2}\right] \times B\left(x_{0}, a R\right), \quad Q_{+}=\left[t_{0}+3 \delta R^{2}, t_{0}+4 \delta R^{2}\right] \times B\left(x_{0}, a R\right)$.

Let $h:\left[t_{0}, \infty\right) \times \mathbb{R}^{d} \rightarrow \mathbb{R}_{+}$be bounded and supported in $\left[t_{0}, \infty\right) \times B\left(x_{0}, 3 a R\right)^{c}$. Then there exists $C_{1}=C_{1}\left(\delta, a, R_{*}\right)>0$ independent of $R \geq R_{*}$ such that the following holds:

$$
\mathbb{E}_{\left(t_{1}, y_{1}\right)}\left[h\left(Z_{\tau_{Q_{1}}}\right)\right] \leq C_{1} \mathbb{E}_{\left(t_{2}, y_{2}\right)}\left[h\left(Z_{\tau_{Q_{2}}}\right)\right] \quad \text { for }\left(t_{1}, y_{1}\right) \in Q_{-} \text {and }\left(t_{2}, y_{2}\right) \in Q_{+} .
$$

Proof. Given (4.16), the proof is similar to that of 11, Lemma 5.3] (with $R^{2}$ in place of $\phi(R)$ there), except with the following changes. In order to estimate $\int_{0}^{t_{1}} I_{2} d s$ in [11. page 1081] from above, we claim that

$$
p^{B_{3 a R / 2}}\left(t_{1}-s, y_{1}, z\right) \leq c_{1} R^{-d} \quad \text { for every } z \in B_{3 a R / 2} \text { and } t_{1}>s .
$$

Note that

$$
a R_{*} / 4 \leq a R / 4 \leq\left|y_{1}-z\right| \leq 5 a R / 2 \quad \text { for every } z \in B_{3 a R / 2} .
$$

If $t_{1}-s \leq 1$, by Theorem 3.6 and (4.19)

$$
p^{B_{3 a R / 2}}\left(t_{1}-s, y_{1}, z\right) \leq p\left(t_{1}-s, y_{1}, z\right) \leq c_{2} \frac{1}{\left|y_{1}-z\right|^{d}} \leq \frac{c_{3}}{R^{d}} .
$$

If $t_{1}-s>1$ and $\left|y_{1}-z\right| \geq\left(t_{1}-s\right)$, then, by Theorem 3.6 and [4.19),

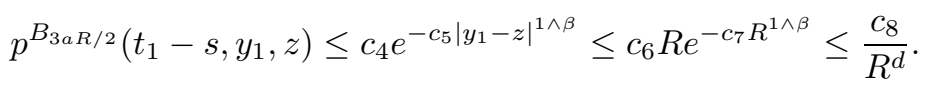

If $t_{1}-s>1$ and $\left|y_{1}-z\right|^{(2-\beta) \vee 1}<\left(t_{1}-s\right)$, then by (4.19), $\frac{a R}{4} \leq\left|y_{1}-z\right|<$ $\left(t_{1}-s\right)^{\frac{1}{(2-\beta) \vee 1}}$. Thus by Theorem 3.6

$p^{B_{3 a R / 2}}\left(t_{1}-s, y_{1}, z\right) \leq c_{9}\left(t_{1}-s\right)^{-d / 2} e^{-c_{10} \frac{\left|y_{1}-z\right|^{2}}{t_{1}-s}} \leq c_{11}(a R / 4)^{-d / 2} e^{-5 c_{10} a R / 2} \leq \frac{c_{12}}{R^{d}}$.

If $t_{1}-s>1, \beta \in(0,1]$ and $\left|y_{1}-z\right|^{2-\beta} \geq\left(t_{1}-s\right) \geq\left|y_{1}-z\right|$, then, by (4.19), $a R / 4 \leq\left|y_{1}-z\right|<t_{1}-s$. Thus by Theorem 3.6(a)

$$
p^{B_{3 a R / 2}}\left(t_{1}-s, y_{1}, z\right) \leq c_{13} e^{-c_{14}\left|y_{1}-z\right|^{\beta}} \leq c_{13} e^{-c_{15} R^{\beta}} \leq \frac{c_{16}}{R^{d}} .
$$

We have proved (4.18), which implies that $\int_{0}^{t_{1}} I_{2} d s$ in [11, page 1081] is less than or equal to the right hand side of [11, (5.4)]. Now using UJS, the remainder of the proof is similar to that of [11, Lemma 5.3]. We omit the rest of the proof.

We say that a non-negative Borel measurable function $h(t, x)$ on $[0, \infty) \times \mathbb{R}^{d}$ is parabolic (or caloric) on $D=(a, b] \times B\left(x_{0}, r\right)$ if for every relatively compact open subset $D_{1}$ of $D, h(t, x)=\mathbb{E}_{(t, x)}\left[h\left(Z_{\tau_{D_{1}}}\right)\right]$ for every $(t, x) \in D_{1} \cap\left([0, \infty) \times \mathbb{R}^{d}\right)$, where $\tau_{D_{1}}=\inf \left\{s>0: Z_{s} \notin D_{1}\right\}$. 
Theorem 4.11. For every $\delta \in(0,1)$ there exists $c=c(\phi, d, \delta, \beta)>0$ such that for every $x_{0} \in \mathbb{R}^{d}, t_{0} \geq 0, R>0$ and every non-negative function $u$ on $[0, \infty) \times \mathbb{R}^{d}$ that is parabolic on $\left(t_{0}, t_{0}+4 \delta\left(\phi_{1}(R) \vee R^{2}\right)\right] \times B\left(x_{0}, 4 R\right)$,

$$
\sup _{\left(t_{1}, y_{1}\right) \in Q_{-}} u\left(t_{1}, y_{1}\right) \leq c \inf _{\left(t_{2}, y_{2}\right) \in Q_{+}} u\left(t_{2}, y_{2}\right)
$$

where $Q_{-}=\left(t_{0}+\delta\left(\phi_{1}(R) \vee R^{2}\right), t_{0}+2 \delta\left(\phi_{1}(R) \vee R^{2}\right)\right] \times B\left(x_{0}, R\right)$ and $Q_{+}=\left[t_{0}+\right.$ $\left.3 \delta\left(\phi_{1}(R) \vee R^{2}\right), t_{0}+4 \delta\left(\phi_{1}(R) \vee R^{2}\right)\right] \times B\left(x_{0}, R\right)$.

Proof. This result was already proved in [11, Theorem 5.2] and [13, Theorem 4.12] for $R \leq R_{0}$. Given the above lemmas, one can prove for the case $R>R_{0}$ similar to the proof in [11, Theorem 5.2] (see also [13, Theorem 4.12]) for $\beta<\infty$, and the proof in [10, Theorem 4.1] for $\beta=\infty$.

\section{OfF-Diagonal LOWER Bound estimates}

For the remainder of this paper, $(\mathcal{E}, \mathcal{F})$ is the Dirichlet form given by (1.8) and (1.10), with the jumping kernel $J$ satisfying conditions (1.1)-(1.4), (1.7) and UJS, and $Y$ is the associated Hunt process on $\mathbb{R}^{d}$. Recall that $\beta>0$ is the exponent in (1.2).

In this section, we give the proof of the off-diagonal lower bound for $p(t, x, y)$. Using the results in previous sections, the proof of the off-diagonal lower bound for the limiting case $\beta=\infty$ (i.e. the case $\psi_{1}(r)=\infty$ for every $r \geq 1$ ) is identical to the one in [10]. Thus in this section we only consider the case $0<\beta<\infty$.

Lemma 5.1. (i) For every $r_{0}>0$ there exist $c_{1}, c_{2} \in(0,1)$ such that for every $x \in \mathbb{R}^{d}$ and $0<r \leq r_{0}$,

$$
\mathbb{P}_{x}\left(\tau_{B(x, r / 2)}<c_{1} \phi_{1}(r)\right) \leq c_{2} .
$$

(ii) There exists $\gamma \in(0,1)$ such that for every $t \geq \frac{1}{4}$ and $x \in \mathbb{R}^{d}$,

$$
\mathbb{P}_{x}\left(\tau_{B\left(x, \gamma^{-1 / 2} t^{1 / 2}\right)}<t\right) \leq \frac{1}{2} .
$$

Proof. Since the idea of the proof is similar, we only spell out details for the proof of (ii) (see also [11, Lemma 2.5] for the proof of (i)).

Let $x \in \mathbb{R}^{d}$ and $t>1$. When $\beta \in(1, \infty)$, by Theorem 3.6 (b),

$$
\begin{aligned}
& \mathbb{P}_{x}\left(Y_{t} \notin B(x, r)\right)=\int_{B(x, r)^{c}} p(t, x, z) d z \\
\leq & c_{1} \int_{\left\{z \in \mathbb{R}^{d}:|z-x| \geq(r \vee t)\right\}} t e^{-c_{2}|z-x|(\log |z-x| / t)^{(\beta-1) / \beta}} d z \\
& \quad+c_{1} \int_{\left\{z \in \mathbb{R}^{d}: t \geq|z-x| \geq r\right\}} t^{-d / 2} \exp \left(-\frac{c_{3}|z-x|^{2}}{t}\right) d z \\
\leq & c_{5} \int_{\left\{w \in \mathbb{R}^{d}:|w| \geq(r \vee t)\right\}} e^{-c_{4}|w|} d w+c_{5} \mathbf{1}_{(0, t]}(r) \int_{r}^{t} t^{-d / 2} \exp \left(-\frac{c_{3} s^{2}}{t}\right) s^{d-1} d s \\
\leq & c_{5} \int_{\left\{w \in \mathbb{R}^{d}:|w| \geq r\right\}} e^{-c_{4}|w|} d w+c_{6} \mathbf{1}_{(0, t]}(r) \int_{r /(\sqrt{t})}^{\infty} \exp \left(-c_{3} u^{2}\right) u^{d-1} d u .
\end{aligned}
$$


When $\beta \in(0,1]$, using Theorem 3.6(a),

$$
\begin{aligned}
& \mathbb{P}_{x}\left(Y_{t} \notin B(x, r)\right) \\
& \leq c_{7} \int_{\left\{z \in \mathbb{R}^{d}:|z-x| \geq\left(r \vee t^{1 /(2-\beta)}\right)\right\}} t e^{-c_{8}|z-x|^{\beta}} d z \\
& +c_{7} \int_{\left\{z \in \mathbb{R}^{d}: t^{1 /(2-\beta)} \geq|z-x| \geq r\right\}} t^{-d / 2} \exp \left(-\frac{c_{9}|z-x|^{2}}{t}\right) d z \\
& \leq c_{10} \int_{\left\{w \in \mathbb{R}^{d}:|w| \geq\left(r \vee t^{1 /(2-\beta)}\right)\right\}} e^{-c_{11}|w|^{\beta}} d w \\
& +c_{10} \mathbf{1}_{\left(0, t^{1 /(2-\beta)}\right]}(r) \int_{r}^{t^{1 /(2-\beta)}} t^{-d / 2} \exp \left(-\frac{c_{9} s^{2}}{t}\right) s^{d-1} d s \\
& \leq c_{10} \int_{\left\{w \in \mathbb{R}^{d}:|w| \geq r\right\}} e^{-c_{11}|w|^{\beta}} d w+c_{12} \mathbf{1}_{\left(0, t^{1 /(2-\beta)}\right]}(r) \int_{r /(\sqrt{t})}^{\infty} \exp \left(-c_{9} u^{2}\right) u^{d-1} d u .
\end{aligned}
$$

Thus for each $\beta>0$ we can choose $\gamma>0$ small so that

$$
\mathbb{P}_{x}\left(Y_{t} \notin B(x, r)\right)<1 / 4 \quad \text { for every } t \leq \gamma r^{2}
$$

for $t \geq \frac{1}{4}$. Using (5.1), we see that (by changing $\gamma$ if necessary) (5.2) holds for all $t>0$. Thus, by [1, Lemma 3.8]), we obtain

$$
\mathbb{P}_{x}\left(\tau_{B(x, 2 r)}<\gamma r^{2}\right)=\mathbb{P}_{x}\left(\sup _{u \leq \gamma r^{2}}\left|Y_{u}-Y_{0}\right|>r\right) \leq \frac{1}{2},
$$

for every $r^{2} \gamma \geq 1$, so the proof of (ii) is completed by considering $t=4 \gamma r^{2}$.

Let $\gamma$ be the constant in Lemma 5.1(ii). Applying Lemma 5.1] and (1.11), we can prove the following in the same way as [13, Proposition 4.11]. Thus we skip the proof.

Proposition 5.2. (i) If $(t, x, y) \in\left[\frac{1}{4}, \infty\right) \times \mathbb{R}^{d} \times \mathbb{R}^{d}$ with $|x-y| \geq 3 \gamma^{-1 / 2} t^{1 / 2}$, then there exist constants $c_{1}, c_{2}>0$ such that

$$
\mathbb{P}_{x}\left(Y_{t} \in B\left(y, 3 \cdot 2^{-1} \gamma^{-1 / 2} t^{1 / 2}\right)\right) \geq c_{1} \frac{t^{1+d / 2} e^{-c_{2}|x-y|^{\beta}}}{|x-y|^{d} \phi_{1}(|x-y|)} .
$$

(ii) For every $t_{0}>0$ there exist constants $c_{1} \geq 2, c_{2}>0$ and $c_{3}>0$ such that for every $x, y \in \mathbb{R}^{d}$ with $|x-y| \geq c_{1} \phi^{-1}(t)$ and for every $t \in\left(0, t_{0}\right]$,

$$
\mathbb{P}_{x}\left(Y_{t} \in B\left(y, c_{1} \phi^{-1}(t)\right)\right) \geq c_{2} \frac{t\left(\phi^{-1}(t)\right)^{d}}{|x-y|^{d} \phi\left(c_{3}|x-y|\right)} .
$$

Now using Theorem 4.11, Lemma 5.1(i) and Proposition 5.2(ii), the proof of the next theorem is almost identical to that of [13, Lemma 4.13 and Theorem 1.2]. (For the case of $|x-y| \leq c$ for some small $c>0$, this has been proved in [11, Theorem 2.4].) Thus we skip its proof.

Theorem 5.3. Suppose $t_{0} \in(0, \infty)$. There exists $c=c\left(\beta, t_{0}\right)>0$ such that for every $t \leq t_{0}$,

$$
p(t, x, y) \geq c\left(\frac{1}{\left(\phi^{-1}(t)\right)^{d}} \wedge \frac{t}{|x-y|^{d} \phi\left(c_{1}|x-y|\right)}\right) .
$$


Theorem 5.4. (i) For every $C^{*}>0$ there exist $c_{1}, c_{2}>0$ such that for every $t \geq 1$,

$$
p(t, x, y) \geq c_{1} t^{-d / 2} \exp \left(-\frac{c_{2}|x-y|^{2}}{t}\right) \quad \text { when } C^{*}|x-y| \leq t .
$$

(ii) There exist $c_{1}, c_{2}>0$ such that for every $t \geq 1$,

$$
p(t, x, y) \geq c_{1} t e^{-c_{2}|x-y|^{\beta}} \quad \text { when }|x-y| \geq \frac{\sqrt{3}}{2} \gamma^{-1 / 2} t^{1 / 2},
$$

where $\gamma$ is the constant in Lemma 5.1(ii).

In particular, when $0<\beta \leq 1$, there exist $c_{1}, c_{2}, C_{*}>0$ such that for every $t \geq 1$,

$$
p(t, x, y) \geq c_{1} t e^{-c_{2}|x-y|^{\beta}} \quad \text { when }|x-y|^{2-\beta} \geq t / C_{*} .
$$

Proof. (i) Fix $C^{*}>0$ and let $R:=|x-y|$. By Theorem 4.8, we only need to consider the case $1 \vee\left(C^{*} R\right) \leq t \leq R^{2}$. Let $l$ be the smallest positive integer so that $t / l \geq(R / l)^{2}$. Then $1 \leq R^{2} / t \leq l<1+R^{2} / t \leq 2 R^{2} / t$, and so $2(R / l)^{2} \geq t / l \geq$ $(R / l)^{2}$. Since $t \geq C^{*} R$,

$$
\frac{t}{l} \geq \frac{t}{1+R^{2} / t}=\frac{t^{2}}{t+R^{2}} \geq 2^{-1}\left(\frac{t}{R}\right)^{2} \geq 2^{-1}\left(C^{*}\right)^{2} .
$$

Let $x=x_{0}, x_{1}, \cdots, x_{l}=y$ be such that $\left|x_{i}-x_{i+1}\right|=R / l$ for $i=1, \cdots, l-1$, and let $B_{i}:=B\left(x_{i}, R / l\right)$. Since $(R / l)^{2} \leq t / l$ and $t / l \geq 2^{-1}\left(C^{*}\right)^{2}$, by Theorem 4.8 and the semigroup property, we have

$$
p\left(t / l, y_{i}, y_{i+1}\right) \geq c_{1}(t / l)^{-d / 2}, \quad \text { for every }\left(y_{i}, y_{i+1}\right) \in B_{i} \times B_{i+1} .
$$

Using (5.5), we have

$$
\begin{aligned}
p(t, x, y) & \geq \int_{B_{1}} \ldots \int_{B_{l-1}} p\left(t / l, x, y_{1}\right) \ldots p\left(t / l, y_{l-1}, y\right) d y_{1} \ldots d y_{l-1} \\
& \geq c_{1}(t / l)^{-d / 2} \Pi_{i=1}^{l-1}\left(c_{2}(t / l)^{-d / 2}(R / l)^{d}\right) \geq c_{3}(t / l)^{-d / 2}\left(c_{2} 2^{-d / 2}\right)^{l-1} \\
& \geq c_{3}(t / l)^{-d / 2} \exp \left(-c_{4} l\right) \geq c_{5} t^{-d / 2} \exp \left(-\frac{c_{6}|x-y|^{2}}{t}\right) .
\end{aligned}
$$

(ii) Since $|x-y| \geq 3 \cdot 2^{-1} \gamma^{-1 / 2}(t / 3)^{1 / 2}$, applying Proposition 5.2 (i) with $t / 3$ in place of $t$, we have

$$
\mathbb{P}_{x}\left(Y_{t / 3} \in B\left(y, C_{1} t^{1 / 2}\right)\right) \geq \mathbb{P}_{x}\left(Y_{t / 3} \in B\left(y, C_{1}(t / 3)^{1 / 2}\right)\right) \geq c_{7} \frac{t^{1+d / 2} e^{-c_{8}|x-y|^{\beta}}}{|x-y|^{d} \phi_{1}(|x-y|)},
$$

where $C_{1}:=3 \cdot 2^{-1} \gamma^{-1 / 2}$. As $\mu_{d}\left(B\left(y, C_{1} t^{1 / 2}\right)\right) \leq c_{9} t^{d / 2}$, the above, together with Theorem 4.11, implies that

$$
p(t, x, y) \geq c_{10} \sup _{w \in B\left(y, C_{1} t^{1 / 2}\right)} p(t / 3, x, w) \geq c_{11} \frac{t e^{-c_{8}|x-y|^{\beta}}}{|x-y|^{d} \phi_{1}(|x-y|)},
$$

which is greater than $c_{12} t e^{-c_{14}|x-y|}$ since $|x-y| \geq \frac{\sqrt{3}}{2} \gamma^{-1 / 2}$. This completes the proof. 
Theorem 5.5. Suppose $\beta>1$ and $C_{*} \in(0,1)$. There exist $c_{1}, c_{2}>0$ depending on $C_{*}$ and $\beta$ such that

$$
p(t, x, y) \geq c_{1} \exp \left(-c_{2}|x-y|\left(\log \frac{|x-y|}{t}\right)^{(\beta-1) / \beta}\right)
$$

for every $t>1$ and $|x-y| \geq t / C_{*}$, and

$$
p(t, x, y) \geq c_{1} t\left(e^{-c_{2}|x-y|\left(\log \frac{|x-y|}{t}\right)^{\frac{\beta-1}{\beta}}}+e^{-c_{2}|x-y|^{\beta}}\right)
$$

for every $t \in(0,1]$ and $|x-y| \geq t / C_{*}$.

Proof. Let $R:=|x-y|$. Note that $\exp \left(-c R(\log (R / t))^{(\beta-1) / \beta}\right) \geq \exp \left(-c R^{\beta}\right)$ is equivalent to $t \geq R \exp \left(-R^{\beta}\right)$. Since $R \exp \left(-R^{\beta}\right) \leq \exp (-1)$ for all $R>0$ and $\beta \geq 1, t \geq R \exp \left(-R^{\beta}\right)$ holds for $t>1$. When $t<R \exp \left(-R^{\beta}\right)$ (which can only occur for some $t \in(0,1])$, (5.7) holds by Theorem 5.3. So it suffices to consider the case $C_{*} R \geq t \geq R \exp \left(-R^{\beta}\right)$. In this case we have $R(\log R / t)^{-1 / \beta} \geq 1$. Let $l \geq 2$ be a positive integer such that

$$
R(\log (R / t))^{-1 / \beta}<l \leq R(\log (R / t))^{-1 / \beta}+1
$$

and let $x=x_{0}, x_{1}, \cdots, x_{l}=y$ be such that $\left|x_{i}-x_{i+1}\right| \leq 2 R / l$ for $i=1, \cdots, l-1$. (Here we used the fact that $\mathbb{R}^{d}$ is a geodesic space.) We observe that

$$
\frac{t}{l} \leq \frac{t}{R}(\log (R / t))^{1 / \beta} \leq \sup _{s \geq 1 / C_{*}} s^{-1}(\log s)^{1 / \beta}=: t_{0}<\infty
$$

and

$$
\frac{R}{2 l} \geq \frac{1}{2}\left(1-\frac{1}{l}\right)(\log (R / t))^{1 / \beta} \geq \frac{1}{4}\left(\log \left(C_{*}^{-1}\right)\right)^{1 / \beta}=: r_{0}>0 .
$$

Thus for all $\left(y_{i}, y_{i+1}\right) \in B\left(x_{i}, r_{0}\right) \times B\left(x_{i+1}, r_{0}\right), 3 R / l \geq\left|y_{i}-y_{i+1}\right| \geq R / l$ and $\phi\left(\left|y_{i}-y_{i+1}\right|\right) \geq \phi(R / l) \geq \phi\left(2 r_{0}\right) \geq\left(\phi\left(2 r_{0}\right) / t_{0}\right)(t / l)$. Now, by Theorem 5.3 with our $t_{0}$, we have for all $\left(y_{i}, y_{i+1}\right) \in B\left(x_{i}, r_{0}\right) \times B\left(x_{i+1}, r_{0}\right)$,

$$
\begin{aligned}
p\left(t / l, y_{i}, y_{i+1}\right) & \geq c_{1} \frac{t / l}{(R / l)^{d} \phi(4 R / l)} \geq c_{2} \frac{t}{R}(l / R)^{d-1+\beta_{2}} e^{-\gamma_{2}(3 R / l)^{\beta}} \\
& \geq c_{3} \frac{t}{R}(\log (r / t))^{-\left(d-1+\beta_{2}\right) / \beta}\left(\frac{t}{R}\right)^{\gamma_{2} 3^{\beta}} \geq c_{4}(t / R)^{c_{5}},
\end{aligned}
$$

where $c_{5}>1$. Let $B_{i}=B\left(x_{i}, r_{0}\right)$. Using (5.8), we have

$$
\begin{aligned}
p(t, x, y) & \geq \int_{B_{1}} \ldots \int_{B_{l-1}} p\left(t / l, x, y_{1}\right) \ldots p\left(t / l, y_{l-1}, y\right) d y_{1} \ldots d y_{l-1} \\
& \geq c_{4}(t / R)^{c_{5}} \Pi_{i=1}^{l-1}\left(c_{4}(t / R)^{c_{5}} \cdot r_{0}^{d}\right) \\
& \geq c_{7} \exp \left(-c_{5}\left(R(\log (R / t))^{-1 / \beta}+1\right) \log \left(c_{8}^{-1} R / t\right)\right) \\
& \geq c_{9} \exp \left(-c_{10} R(\log (R / t))^{1-1 / \beta}\right) .
\end{aligned}
$$

If in addition $t \in(0,1]$, then

$$
\exp \left(-c_{10} R(\log (R / t))^{1-1 / \beta}\right) \geq t \exp \left(-c_{10} R(\log (R / t))^{1-1 / \beta}\right) .
$$

We thus obtain (5.6) and (5.7).

Combining Theorems 5.35 .5 , we have the lower bounds in Theorem 1.2 . 


\section{Applications of heat Kernel estimates}

6.1. Green function estimates. We assume $d \geq 3$ and give two-sided sharp estimates the Green function for $G(x, y)$ of $Y$ in $\mathbb{R}^{d}$ where

$$
G(x, y):=\int_{0}^{\infty} p(t, x, y) d t, \quad x, y \in \mathbb{R}^{d} .
$$

Theorem 6.1. There exists $c=c(\alpha, d)>1$ such that for $x, y \in \mathbb{R}^{d}$,

$$
c^{-1}\left(\frac{\phi_{1}(|x-y|)}{|x-y|^{d}}+\frac{1}{|x-y|^{d-2}}\right) \leq G(x, y) \leq c\left(\frac{\phi_{1}(|x-y|)}{|x-y|^{d}}+\frac{1}{|x-y|^{d-2}}\right) .
$$

Proof. Note that for every $T, M \in[0, \infty)$

$$
\int_{T}^{\infty} t^{-\frac{d}{2}} e^{-\frac{M|x-y|^{2}}{t}} d t=\frac{1}{|x-y|^{d-2}} \int_{0}^{\frac{|x-y|^{2}}{T}} u^{\frac{d-4}{2}} e^{-M u} d u
$$

When $|x-y| \leq 1$, by Theorem 1.2 and (6.1),

$$
\begin{aligned}
& c_{1} \frac{\phi_{1}(|x-y|)}{|x-y|^{d}} \leq c_{2} \int_{0}^{\phi_{1}(|x-y|) \wedge 1} \frac{t}{|x-y|^{d} \phi\left(c_{1}|x-y|\right)} d t \leq G(x, y) \\
\leq & c_{3} \int_{0}^{\phi_{1}(|x-y|) \wedge 1} \frac{t}{|x-y|^{d} \phi_{1}(|x-y|)} d t \\
& +c_{4} \int_{\phi_{1}(|x-y|) \wedge 1}^{1} \frac{1}{\left(\phi_{1}^{-1}(t)\right)^{d}} d t+c_{5} \int_{1}^{\infty} t^{-\frac{d}{2}} e^{-\frac{c_{6}|x-y|^{2}}{t}} d t \\
\leq & c_{7} \frac{\phi_{1}(|x-y|)}{|x-y|^{d}}+c_{8} \frac{\phi_{1}(|x-y|)}{|x-y|^{d}} \sum_{k=0}^{\infty} \int_{c_{9} \phi_{1}\left(2^{k}|x-y|\right)}^{c_{9} \phi_{1}\left(2^{k+1}|x-y|\right)} \frac{|x-y|^{d}}{\phi_{1}(|x-y|)\left(\phi_{1}^{-1}(t)\right)^{d}} d t \\
& +c_{5} \frac{1}{|x-y|^{d-2}} \int_{0}^{|x-y|^{2}} u^{\frac{d-4}{2}} e^{-c_{6} u^{\beta}} d u \leq c_{10}\left(\frac{\phi_{1}(|x-y|)}{|x-y|^{d}}+\frac{1}{|x-y|^{d-2}}\right),
\end{aligned}
$$

where we used (1.3) in the last inequality. In fact,

$$
\sum_{k=0}^{\infty} \int_{c_{9} \phi_{1}\left(2^{k}|x-y|\right)}^{c_{9} \phi_{1}\left(2^{k+1}|x-y|\right)} \frac{|x-y|^{d}}{\phi_{1}(|x-y|)\left(\phi_{1}^{-1}(t)\right)^{d}} d t \leq c_{11} \sum_{k=0}^{\infty} 2^{-\left(d-\beta_{2}\right) k}<\infty .
$$

On the other hand, if $|x-y|>1$, using Theorems 1.2 and 5.4(ii) and (6.1),

$$
\begin{aligned}
& \frac{c_{12}}{|x-y|^{d-2}} \int_{0}^{1} u^{\frac{d-4}{2}} d u=c_{12} \int_{|x-y|^{2}}^{\infty} t^{-\frac{d}{2}} d t \leq G(x, y) \\
\leq & c_{13} \int_{0}^{1} t e^{-c_{14}|x-y|^{\beta \wedge 1}} d t+c_{15} \int_{1}^{|x-y|^{2-(\beta \wedge 1)}} t e^{-c_{16}|x-y|^{\beta \wedge 1}} d t \\
& +c_{17} \int_{|x-y|^{2-(\beta \wedge 1)}}^{\infty} t^{-\frac{d}{2}} e^{-\frac{c_{18}|x-y|^{2}}{t}} d t \\
\leq & c_{19}|x-y| e^{-c_{20}|x-y|^{\beta \wedge 1}}+\frac{c_{21}}{|x-y|^{d-2}} \int_{0}^{|x-y|^{\beta \wedge 1}} u^{\frac{d-4}{2}} e^{-c_{22} u} d u \leq \frac{c_{23}}{|x-y|^{d-2}} .
\end{aligned}
$$

This completes the proof of the theorem. 
6.2. Differentiability of spectral functions. In [24, 25], the differentiability of spectral functions for symmetric stable processes are studied.

Let $Z$ be a symmetric jump process considered in this paper whose Dirichlet form $(\mathcal{Q}, \mathcal{D})$ is given by (1.8)-(1.9) whose jumping intensity kernel $J$ is given by (1.6) and satisfies UJS. Let $\mu$ be a signed measure in the Kato class $\mathbf{K}_{\infty}(X)$ as introduced in 9 . The associated spectral function $C(\lambda)$ is defined to be

$$
C(\lambda)=-\inf \left\{\mathcal{Q}(u, u)+\lambda \int_{\mathbb{R}^{d}} u(x)^{2} \mu(d x): u \in \mathcal{D} \text { with } \int_{\mathbb{R}^{d}} u(x)^{2} d x=1\right\} .
$$

It follows from [15] and Theorem 6.1 that the extended Dirichlet form $\left(\mathcal{Q}, \mathcal{D}_{e}\right)$ is compactly embedded into $L^{2}\left(\mathbb{R}^{d},|\mu|\right)$. Hence using the heat kernel estimates established in this paper, by an argument almost the same as that in [24, 25], it can be shown that if $d \leq 4$, then $\lambda \mapsto C(\lambda)$ is differentiable on $\mathbb{R}$. But we will not go into details about it here. See [15] for recent progress on the differentiability of spectral functions.

\section{ACKNOWLEDGMENT}

The second-named author thanks Martin Barlow for discussions on UJS that led to Example 2.4

\section{REFERENCES}

1. M.T. Barlow, R.F. Bass, Z.-Q. Chen and M. Kassmann. Non-local Dirichlet forms and symmetric jump processes. Trans. Amer. Math. Soc. 361 (2009), 1963-1999. MR2465826 (2010e:60163)

2. M.T. Barlow, R.F. Bass and T. Kumagai. Parabolic Harnack inequality and heat kernel estimates for random walks with long range jumps. Math. Z. 261 (2009), 297-320. MR2457301 (2009m:60111)

3. M.T. Barlow, A. Grigor'yan and T. Kumagai. Heat kernel upper bounds for jump processes and the first exit time. J. Reine Angew. Math. 626 (2009), 135-157. MR.2492992 (2009m:58077)

4. R. F. Bass and D. A. Levin. Transition probabilities for symmetric jump processes. Trans. Amer. Math. Soc. 354 (2002), 2933-2953. MR.1895210 (2002m:60132)

5. L. A. Caffarelli, S. Salsa and Luis Silvestre. Regularity estimates for the solution and the free boundary to the obstacle problem for the fractional Laplacian. Invent. Math. 171(1) (2008), 425-461. MR2367025 (2009g:35347)

6. E.A. Carlen, S. Kusuoka and D.W. Stroock. Upper bounds for symmetric Markov transition functions. Ann. Inst. H. Poincaré-Probab. Statist. 23 (1987), 245-287. MR898496 (88i:35066)

7. R. Carmona, W. C. Masters and B. Simon. Relativistic Schrödinger operators: Asymptotic behavior of the eigenfunctions. J. Funct. Anal. 91(1) (1990), 117-142. MR.1054115 (91i:35139)

8. P. Carr, H. Geman, D. Madan and M. Yor. The Fine Structure of Asset Returns: An Empirical Investigation, Journal of Business, 75(2) (2002), 305-332.

9. Z.-Q. Chen. Gaugeability and conditional gaugeability. Trans. Amer. Math. Soc. 354 (2002), 4639-4679. MR1926893 (2003i:60127)

10. Z.-Q. Chen, P. Kim and T. Kumagai. Weighted Poincaré inequality and heat kernel estimates for finite range jump processes. Math. Ann. 342(4) (2008), 833-883 MR2443765 (2010b:60230)

11. Z.-Q. Chen, P. Kim and T. Kumagai. On heat kernel estimates and parabolic Harnack inequality for jump processes on metric measure spaces. Acta Math. Sin. (Engl. Ser.) 25 (2009), 1067-1086. MR2524930

12. Z.-Q. Chen and T. Kumagai. Heat kernel estimates for stable-like processes on $d$-sets. Stochastic Process Appl. 108 (2003), 27-62. MR.2008600 (2005d:60135)

13. Z.-Q. Chen and T. Kumagai. Heat kernel estimates for jump processes of mixed types on metric measure spaces. Probab. Theory Relat. Fields 140 (2008), 277-317. MR.2357678(2009e:60186) 
14. Z.-Q. Chen and R. Song. Drift transforms and Green function estimates for discontinuous processes. J. Funct. Anal. 201 (2003), 262-281. MR.1986161 (2004c:60218)

15. Z.-Q. Chen and K. Tsuchida. Large deviation, compact embedding and differentiability of spectral functions. In preparation.

16. M. Fukushima, Y. Oshima and M. Takeda. Dirichlet Forms and Symmetric Markov Processes. de Gruyter, Berlin, 1994. MR1303354 (96f:60126)

17. I. Koponen, Analytic approach to the problem of convergence of truncated Levy flights towards the Gaussian stochastic process, Physical Review E, 52 (1995), 1197-1199.

18. E. H. Lieb and H.-T. Yau. The stability and instability of relativistic matter. Comm. Math. Phys. 118(2) (1988), 177-213. MR956165(90c:81251)

19. R. N. Mantegna and H. E. Stanley. Stochastic processes with ultraslow convergence to a Gassuan: The truncated Lévy flight. Phys. Rev. Letter 73 (1994), 2946-2949. MR.1303317 (95g:82062)

20. J. Masamune and T. Uemura. Conservation property of symmetric jump processes. To appear in Ann. Inst. Henri Poincaré Probab. Stat.

21. A. Matacz. Financial modeling and option theory with the truncated Lévy process. Int. J. Theor. Appl. Finance 3(1) (2000), 143-160.

22. P.-A. Meyer. Renaissance, recollements, mélanges, ralentissement de processus de Markov. Ann. Inst. Fourier 25 (1975), 464-497. MR0415784 (54:3862)

23. J. Rosiński. Tempering stable processes. Stochastic Process. Appl. 117(6) (2007), 677-707. MR:2327834 (2008g:60146)

24. M. Takeda and K. Tsuchida. Differentiability of spectral functions for symmetric $\alpha$-stable processes. Trans. Amer. Math. Soc. 359 (2007), 4031-4054. MR.2302522 (2008a:35045)

25. K. Tsuchida. Differentiability of spectral functions for relativistic $\alpha$-stable processes with application to large deviations. Potential Anal. 28 (2008), 17-33. MR2366397(2008j:60191)

Department of Mathematics, University of Washington, Seattle, Washington 98195

E-mail address: zchen@math.washington.edu

Department of Mathematical Science, Seoul National University, Seoul 151-747, SOUTH KoreA

E-mail address: pkim@snu.ac.kr

Research Institute for Mathematical Sciences, Kyoto University, Kyoto 606-8502, JAPAN

E-mail address: kumagai@kurims.kyoto-u.ac.jp 